\title{
APPLICATIONS OF BANACH IDEALS OF OPERATORS
}

\author{
BY J. R. RETHERFORD ${ }^{1,2}$

\section{For Stacy and Dana}

1. Apologies. Since much of the recent work in the Banach space aspects of Functional Analysis, especially the geometry of Banach spaces, could, by a bit of chicanery, be construed as applications of Banach ideals, the title does not indicate a complete survey. This work is surely not exhaustive of the subject matter. Thus, many good papers are totally ignored. This is somewhat compensated for by the monograph [1.1] of Lindenstrauss and Tzafriri on the geometry of the classical Banach spaces and the "pre-book" [1.2] of A. Pietsch on the general theory of ideals of operators.

Since a lecture should have a central theme, I have chosen a fundamental result of Grothendieck which asserts that there are Banach spaces $E$ and $F$ for which every bounded linear operator from $E$ to $F$ is 2-absolutely summing. (Definitions will be forthcoming.) This result and the local structure of Banach spaces are the unifying topics of this paper. For the numerous topics this unification omits, again, apologies.

I have addressed myself to the material at hand twice before [1.3], [1.4], [1.5], the latter in collaboration with Y. Gordon and D. R. Lewis. I apologize for mentioning, again, the beautiful result of Stegall and Lewis $[1.6]$ and tramping once again over ground covered in [1.3] $-[1.5]$. However, I feel, perhaps with prejudice, that these results are worthy of further discussion.

An additional apology of sorts is needed. I have included many definitions which are old hat to experts in Banach space theory. It is hoped that the material will thus be accessible to a larger audience, perhaps even to some persons completely outside Functional Analysis.

Finally, many of the results stated have their natural setting in Probability Theory. I have avoided the probabilistic language entirely. Thus "random variable" becomes "measurable function" etc. This is an editorial judgment on my part, and apologies are extended to anyone this may offend.

This is an expanded version of an invited address given at the meeting of the Society in Nashville, Tennessee, November 8, 1974.

AMS (MOS) subject classifications (1970). Primary 46-02, 47-02, 46C05, 46B10, 46B15, 46E05, 46E15, 46E30, 47B10.

Key words and phrases. Banach ideals of operators, type, cotype, stability, LUST, pabsolutely summing, nuclear, integral, $\mathfrak{S}_{\mathrm{p}}-, \mathfrak{D}_{\mathrm{p}}$ - and $\mathscr{S}_{\mathrm{p}}$-spaces.

${ }^{1}$ This paper was prepared under grant GP-34193 of the National Science Foundation (U.S.A.).

${ }^{2}$ Portions of this paper were written during a visit to the Institut für Angewandte Mathematik der Universität Bonn. The author gratefully acknowledges partial support from the Sonderforschungsbereich 72 . 
A remark on the Bibliography is in order. Instead of listing bibliographical references alphabetically we have listed the results as they appear in the text, e.g. [5.3] means reference [3] of \$5. Thus, one interested in the proof of results stated in a given section can go to the source immediately. Due to the length of the paper almost all proofs are omitted; hopefully, this unorthodox bibliography will help compensate for that.

2. Notation. Most of our notation is standard. All spaces considered are Banach spaces. The word operator means bounded linear transformation. By an isomorphism we mean a one-to-one, open operator. The Banach-Mazur distance, $d(E, F)$, between Banach spaces $E$ and $F$, is given by

$$
d(E, F)=\inf \|T\|\left\|T^{-1}\right\|
$$

where the infimum is taken over all isomorphisms between $E$ and $F$. If $E$ and $F$ are not isomorphic set $d(E, F)=+\infty$.

A projection $P$ is an operator from $E$ to $E$ with $P^{2}=P$. If $A$ is a subspace (=closed linear manifold) then $A$ is complemented in $E$ if there is a projection $P$ with $P(E)=A$. We will denote the identity operator on a Banach space $E$ by $\mathrm{id}_{E}$.

A sequence $\left(x_{n}\right)$ in a Banach space $E$ is a Schauder basis for $E$ (unconditional Schauder basis for $E$ ) if for each $x \in E$ there is a unique sequence of scalars $\left(a_{n}\right)$ such that $\sum_{n=1}^{\infty} a_{n} x_{n}$ converges to $x$ in norm (such that $\sum_{n=1}^{\infty} \varepsilon_{n} a_{n} x_{n}$ converges for all choices of signs $\left.\varepsilon_{n}= \pm 1\right)$. The functionals $f_{i}$, defined by $f_{i}(x)=a_{i}$, are called the coefficient functionals of the basis $\left(x_{i}\right)$.

For $1 \leqq p \leqq \infty$ we denote by $l_{p}$ the Banach space of scalar sequences $\left(a_{i}\right)$ with

$$
\begin{aligned}
& \|a\|=\left(\sum_{i=1}^{\infty}\left|a_{i}\right|^{p}\right)^{1 / p} \quad \text { if } \quad 1 \leqq p<\infty, \\
& =\sup _{i}\left|a_{i}\right| \quad \text { if } \quad p=\infty .
\end{aligned}
$$

Similarly, for $l_{p}(\Gamma)$ where $\Gamma$ is any discrete set. In particular, we denote by $l_{p}^{n}$ the space of $n$-tuples with the above norm. We denote by $c_{0}$ the closed subspace of $l_{\infty}$ consisting of those sequences which tend to 0 . Given $p$ in $[1, \infty)$ we will always denote by $p^{\prime}$ the number satisfying $1 / p+1 / p^{\prime}=1$.

If $E^{\prime}$ denotes the conjugate of a Banach space $E$ then $\left(l_{p}\right)^{\prime}=l_{p^{\prime}}$.

We will use tensor notation in $\$ \S 11,12$, and 14 . By $l_{p} \hat{\otimes} l_{q}$ we mean the closure of the finite rank operators $T: l_{p^{\prime}} \rightarrow l_{q}$ in the norm $\inf \left\{\sum_{i=1}^{n}\left\|f_{i}\right\|\left\|x_{i}\right\|: T x=\sum_{i=1}^{n} f_{i}(x) x_{i}\right\}$. By $l_{p} \hat{\otimes} l_{q}$ we mean the closure of the same finite rank operators in the usual operator norm. A similar statement holds for $l_{p}^{n} \otimes l_{q}^{n}$ and $l_{p}^{n} \otimes l_{q}^{n}$.

If $(S, \Sigma, \mu)$ is a measure space then as usual $L_{p}(S, \Sigma, \mu)$ or $L_{p}(\mu)$ denotes the Banach space of equivalence classes of almost everywhere equal functions under the norm

$$
\begin{array}{rlrl}
\|f\|_{p} & =\left(\int_{s}|f(s)|^{p} \mu(d s)\right)^{1 / p} & \text { for } & 1 \leqq p<\infty, \\
& =\underset{s \in S}{\operatorname{ess~uup}}|f(s)| & \text { for } \quad p=\infty
\end{array}
$$


By a probability measure space $(\Omega, \mu)$ we mean a positive measure with $\mu(\Omega)=1$. For $\mu$ Lebesgue measure on $[0,1]$ we will suppress $\mu$ (or $d t)$ and write $L_{p}[0,1]$.

By $C(K)$ we mean the Banach space of continuous scalar valued functions under the sup norm.

If $\left(E_{n}\right)$ is a sequence of Banach spaces then

$$
\left(\bigoplus E_{n}\right) l_{p}=\left\{\left(x_{n}\right), x_{n} \in E_{n} \mid\left\|\left(x_{n}\right)\right\|^{p}=\sum_{n=1}^{\infty}\left\|x_{n}\right\|^{p}<+\infty\right\} .
$$

For a Banach space $E$ let

$$
\delta_{E}(t)=\inf \left\{1-\frac{1}{2}\|x+y\|:\|x\|=\|y\|=1,\|x-y\| \geqq t>0\right\} .
$$

The function $\delta_{E}$ is called the modulus of convexity of $E$. If $\delta_{E}(t)>0$ for $0<\varepsilon \leqq 2$ then $E$ is said to be uniformly convex. The spaces $L_{p}(\mu), 1<p<\infty$, are uniformly convex.

Let $\lambda \geqq 1$ and $1 \leqq p \leqq \infty$. A Banach space $E$ is an $\mathscr{L}_{p, \lambda}$-space if for each finite dimensional subspace $F \subset E$ there is a finite dimensional subspace $B$ with $F \subset B \subset E$ such that $d\left(B, l_{p}^{n}\right) \leqq \lambda$ where $n=\operatorname{dim} B$. A space $E$ is an $\mathscr{L}_{\mathrm{p}}$-space if it is an $\mathscr{L}_{\mathrm{p}, \lambda}$-space for some $\lambda \geqq 1$. These spaces, introduced in [2.1], generalize and include the $L_{p}(S, \Sigma, \mu)$ and $C(K)$-spaces above.

An operator $T$ from $E$ to $F$ is compact if the image of the unit ball of $E$ is relatively compact in $F$.

A space $E$ has the approximation property if every compact operator from $F$ to $E$ is the limit of finite rank operators. The space $E$ has the bounded approximation property if there is a constant $C>0$ such that if $B$ is a finite dimensional subspace of $E$ there is an operator on $E$ with finite dimensional range such that $\|T\| \leqq C$ and $T$ restricted to $B$ is the identity. If $C$ can be taken to be 1 then $E$ is said to have the metric approximation property. A remarkable result of Enflo [2.2] (see also [2.3]) asserts that not every Banach space has the approximation property.

A sequence of subspaces $\left(E_{n}\right)$ in a Banach space $E$ is uniformly complemented if there is an $M>0$ and a sequence of projections $\left(P_{n}\right)$ with $P_{n}(E)=E_{n}$ and $\left\|P_{n}\right\| \leqq M$ for all positive integers $n$.

Following [2.4] we will say that a Banach space $E$ is an $\mathscr{S}_{\mathrm{p}, \mathrm{\lambda}}$-space if for each $n E$ contains subspaces $E_{n}$ which are uniformly complemented and $d\left(E_{n}, l_{p}^{n}\right) \leqq \lambda$; and $E$ is a $\mathscr{D}_{p, \lambda}$-space if for each $n E$ contains $E_{n}$ with $d\left(E_{n}, l_{p}^{n}\right) \leqq \lambda$. Finally, $E$ is an $\mathscr{S}_{p}$ - or a $\mathscr{D}_{p}$-space if it is a $\mathscr{D}_{\mathrm{p}, \lambda}$ - or $\mathscr{F}_{\text {-space }}$ for some $\lambda \geqq 1$. For the relationships between $\mathscr{L}_{p}^{-1}-, \mathscr{S}_{p}$ - and $\mathscr{D}_{p}$-spaces we refer the reader to [2.4].

3. History. An ideal in the ring $\mathscr{L}(H)$ of all bounded linear operators on a separable infinite dimensional Hilbert space is a subset $A(H)$ with the properties: if $S, S_{1}, S_{2} \in A(H)$ and $R, T \in \mathscr{L}(H)$ then $S_{1}+S_{2} \in A(H)$ and $R S T \in A(H)$.

The oldest ideal known to the author is the ideal $\sigma_{2}(H)$ of "HilbertSchmidt" operators. This ideal originated with the work of D. Hilbert [3.1] and E. Schmidt [3.2].

In considering the question "What operators on Hilbert space have a 
trace?", F. J. Murray and J. von Neumann [3.3] found the ideal $\sigma_{1}(H)$ of "trace class" operators. Later J. von Neumann and R. Schatten [3.4] generalized the Hilbert-Schmidt and trace class operators to the ideals $\sigma_{p}(H)(0<p<\infty)$. We will have more to say about these important ideals later.

The final work in the ideal theory in $\mathscr{L}(H)$, in the sense that a "complete" characterization of all two-sided ideals was given, was done by J. W. Calkin [3.5]. In particular, Calkin showed that there is a one-to-one correspondence between the ideals $A(H)$ and the permutation invariant ideals in the ring $l_{\infty}$ of bounded sequences. (Further results along these lines were obtained by Schatten [3.6] and Gohberg and Krein [3.7]. More recently, the situation on nonseparable Hilbert spaces has been considered by Oosterbrink [3.8] and his colleagues.)

On the other hand, as we will see, to obtain an ideal theory on Banach spaces that is suitable for applications, it is not sufficient to consider only the ring $\mathscr{L}(E)$ of bounded linear operators on a Banach space $E$. We must consider the space $\mathscr{L}(E, F)$ of bounded linear operators between arbitrary Banach spaces $E$ and $F$.

Roughly speaking, a subset $\mathscr{A}$ of the class $\mathscr{L}$ of all bounded linear operators between all Banach spaces is an ideal if whenever $S, S_{1}, S_{2} \in \mathbb{A}$ and $R, T \in \mathscr{L}$, then $S_{1}+S_{2} \in \mathscr{A}$ and $R S T \in \mathscr{A}$ (whenever $S_{1}+S_{2}$ and $R S T$ are defined). We will give precise definitions later.

The ideal of compact operators was introduced by F. Riesz [3.9] in 1918. This is the first example of a "Banach" ideal known to the author. (Recall that Banach spaces were not introduced until circa 1932!) Still other examples are the ideals of weakly compact operators (S. Kakutani [3.10]), nuclear operators (A. F. Ruston [3.11], A. Grothendieck [3.12]), and the strictly singular operators (T. Kato [3.13]).

Other special classes of operators were considered by numerous authors. However, the general theory of Banach ideals of operators began, I believe, with the fundamental work on tensor products of Schatten [3.14] and Grothendieck [3.12]. However, the nontrivial translation from the language of tensor products to operators on Banach spaces was accomplished in a series of papers by A. Pietsch [3.15]-[3.21] and, in particular, [3.22] which influenced much of the subsequent work in the area.

4. Diversity of applications. The applications of the theory of Banach ideals have been numerous but mainly in three directions:

I-Classifying types of locally convex spaces (e.g. Schwartz spaces, nuclear spaces); papers related to applications of type I include [4.1]-[4.4] and the numerous references given in [4.3] and [4.4]. II-Measure theory on Banach spaces (linear stochastic processes); here the work is mainly by L. Schwartz and the French school. Principal works are [4.5]-[4.7]. See also [4.8] and the Seminaire Maurey-Schwartz 1972-1973, 1973-1974. We will have something to say about the important Schwartz duality theorem later. III-The structure theory of Banach spaces; applications of type III will be our 
concern in this paper. Appropriate references will be given in the subsequent sections.

5. Banach ideals. Throughout the remainder of the paper $\mathscr{L}$ denotes the class of all bounded linear operators between arbitrary Banach spaces and $\mathscr{L}(E, F)$ the set of all such operators between specific Banach spaces $E$ and $F$. We now define an ideal in the sense of A. Pietsch [5.1]. We say that a class $A$ of bounded linear operators is an ideal if for each set $A(E, F)=$ $A \cap \mathscr{L}(E, F)$ one has

(a) if $x^{\prime} \in E^{\prime}, y \in F$ then $x^{\prime} \otimes y \in A(E, F) \quad\left(x^{\prime} \otimes y\right.$ denotes the rank one operator given by $x^{\prime} \otimes y(x)=\left\langle x, x^{\prime}\right\rangle y$. Clearly, every rank one operator has this form.);

(b) $A(E, F)$ is a linear subset of $\mathscr{L}(E, F)$ for each $E$ and $F$; and

(c) if $U \in \mathscr{L}(X, E), T \in A(E, F), V \in \mathscr{L}(F, Y)$, then $V T U \in A(X, Y)$.

The finite rank operators $\mathscr{F}$ obviously form the smallest ideal.

A function $\alpha$ on the operators $T$ in an ideal $A$ to the nonnegative real numbers is an ideal norm if

(d) $x^{\prime} \in E^{\prime}, y \in F$ then $\alpha\left(x^{\prime} \otimes y\right)=\left\|x^{\prime}\right\|\|y\|$;

(e) $S, T \in A(E, F)$ then $\alpha(S+T) \leqq \alpha(S)+\alpha(T)$; and

(f) if $U \in \mathscr{L}(X, E) ; T \in A(E, F)$ and $V \in \mathscr{L}(F, Y)$, then

$$
\alpha(V T U) \leqq\|V\| \alpha(T)\|U\| \text {. }
$$

An ideal $A$ with norm $\alpha,[A, \alpha]$, is a Banach ideal if each component $A(E, F)$ is a Banach space under $\alpha$.

To any linear normed ideal $[A, \alpha]$ one can associate three normed ideals in a more or less natural fashion:

(I) The dual ideal $\left[A^{\prime}, \alpha^{\prime}\right]$ : An operator $T$ is in $A^{\prime}(E, F)$ if and only if $T^{\prime} \in A\left(F^{\prime}, E^{\prime}\right)$. Here $\alpha^{\prime}(T)=\alpha\left(T^{\prime}\right)$;

(II) The conjugate ideal $\left[A^{\Delta}, \alpha^{\Delta}\right]: A^{\Delta}(E, F)$ is the class of all operators $T \in \mathscr{L}(E, F)$ for which there is a $\rho>0$ such that for any $L \in \mathscr{F}(F, E)$

$$
\mid \text { trace } L T \mid \leqq \rho \alpha(L) \text {. }
$$

The norm $\alpha^{\Delta}(T)$ is defined by inf $\rho, \rho$ satisfying the above inequality. $\left[A^{\Delta}, \alpha^{\Delta}\right]$ is always a Banach ideal.

(III) The adjoint ideal $\left[A^{*}, \alpha^{*}\right]: A^{*}(E, F)$ is the class of all $T \in \mathscr{L}(E, F)$ for which there is a $\rho>0$ such that for all finite dimensional Banach spaces $X, Y$ and for all $V \in \mathscr{L}(X, E), U \in A(Y, X), W \in \mathscr{L}(F, Y)$,

$$
\mid \text { trace } V U W T \mid \leqq \rho\|W\|\|V\| \alpha(U) \text {. }
$$

Here the norm $\alpha^{*}$ is also given by inf $\rho, \rho$ satisfying the above inequality. The ideal $\left[A^{*}, \alpha^{*}\right]$ is also always a Banach ideal.

The ideals $A^{\Delta}$ and $A^{*}$ are intimately related. Indeed, for any $T \in \mathscr{L}(E, F)$, $\alpha^{*}(T) \leqq \alpha^{\Delta}(T)$ and equality holds if both $E$ and $F$ have the metric approximation property.

We now give a few examples of Banach ideals. These ideals are due to several different authors. Bibliographical information and a table showing the relationships between various ideals is given in [5.2]. 
(1) Let $C(E, F)$ denote the closure of $\mathscr{F}(E, F)$ in $\mathscr{L}(E, F)$, and $K(E, F)$ the compact operators from $E$ to $F$. Then $[\mathscr{L},\|\cdot\|],[K,\|\cdot\|]$ and $[C,\|\cdot\|]$ are Banach ideals. let

For a finite or denumerably infinite set $\left\{x_{1}, \cdots, x_{N}\right\}$ in a Banach space $E$,

$$
\begin{gathered}
\varepsilon_{p}\left(\left\{x_{i}\right\}=\sup \left\{\left(\sum_{i=1}^{N}\left|\left\langle x_{i}, f\right\rangle\right|^{p}\right)^{1 / p}:\|f\|=1\right\}, \text { if } 1 \leqq p<+\infty,\right. \\
\varepsilon_{\infty}\left(\left\{x_{i}\right\}\right)=\sup \left\{\sup _{i \leqq N}\left|\left\langle x_{i}, f\right\rangle\right|:\|f\|=1\right\} ; \\
\alpha_{p}\left(\left\{x_{i}\right\}\right)=\left(\sum_{i=1}^{N}\left\|x_{i}\right\|^{p}\right)^{1 / p} \text { if } 1 \leqq p<+\infty, \\
\alpha_{\infty}\left(\left\{x_{i}\right\}\right)=\sup _{i \leqq N}\left\|x_{i}\right\| ;
\end{gathered}
$$

and

$$
\sigma_{p}\left(\left\{x_{i}\right\}\right)=\sup \left\{\left|\sum_{i=1}^{N}\left\langle x_{i}, f_{i}\right\rangle\right|: \varepsilon_{p^{\prime}}\left(\left\{f_{i}\right\}\right) \leqq 1\right\}, \quad \frac{1}{p}+\frac{1}{p^{\prime}}=1 .
$$

(2) $\left[\Pi_{p}, \pi_{p}\right]$ denotes the ideal of $p$-absolutely summing operators: $T \in$ $\Pi_{p}(E, F)$ if there is a $\rho>0$ such that $\alpha_{p}\left(\left\{T x_{i}\right\}\right) \leqq \rho \varepsilon_{p}\left(\left\{x_{i}\right\}\right)$, for all finite sets $\left\{x_{1}, \cdots, x_{N}\right\}$ in $E$. The norm $\pi_{p}$ is given by $\pi_{p}(T)=\inf \rho, \rho$ as above.

The ideal $\left[\Pi_{p}, \pi_{p}\right]$ will be extensively used throughout the remainder of the paper.

(3) $\left[D_{p}, d_{p}\right]$ denotes the ideal of strongly $p$-summing operators: $T \in$ $D_{p}(E, F)$ if there is a $\rho>0$ such that $\sigma_{p}\left[\left\{T x_{i}\right\}\right) \leqq \rho \alpha_{p}\left(\left\{x_{i}\right\}\right)$ for all finite sets $\left\{x_{1}, \cdots, x_{N}\right\}$ in $E ; d_{p}(T)=\inf \rho$.

(4) $\left[I_{p}, i_{p}\right]$ denotes the ideal of $p$-integral operators: $T \in I_{p}(E, F)$ if there is a probability measure $\mu$ and operators $V \in \mathscr{L}\left(E, L_{\infty}(\mu)\right)$ and $W \in \mathscr{L}\left(L_{\infty}(\mu), F^{\prime \prime}\right)$, $F^{\prime \prime}$ the bidual of $F$, such that $W j V=i T$, where $j$ is the canonical injection of $L_{\infty}(\mu)$ into $L_{p}(\mu)$ and $i$ the canonical injection of $F$ into $F^{\prime \prime}$, i.e.,

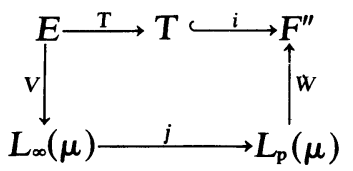

The norm $i_{p}$ is given by $i_{p}(T)=\inf \|V\|\|W\|$, the infimum taken over all probability measures $\mu$ and operators $V, W$.

(5) $\left[N_{p}, \nu_{p}\right]$ denotes the ideal of $p$-nuclear operators: $T \in N_{p}(E, F)$ if $T$ has a representation $T=\sum_{i=1}^{\infty} f_{i} \otimes y_{i}, \quad f_{i} \in E^{\prime}, \quad y_{i} \in F$ and $\alpha_{p}\left(\left\{f_{i}\right\}\right)<+\infty$, and $\varepsilon_{p^{\prime}}\left(\left\{y_{i}\right\}\right)<+\infty\left(1 / p+1 / p^{\prime}=1\right)$. If $p=\infty$ there is the additional requirement that $f_{i} \rightarrow 0$ as $i \rightarrow \infty$. The $p$-nuclear norm is given by $\nu_{p}(T)=\inf \alpha_{p}\left(\left\{f_{i}\right\}\right) \varepsilon_{p^{\prime}}\left(\left\{y_{i}\right\}\right)$, where the infimum is taken over all such representations of $T$.

An operator $T$ in the class $N_{1}$ will be called a nuclear operator. This class of course generalizes the "trace class operators" on Hilbert space.

(6) $\left[C_{p}, c_{p}\right]$ denotes the ideal of operators factoring compactly through $l_{p}: T \in C_{p}(E, F)$ if there are $A \in C\left(E, l_{p}\right), B \in C\left(l_{p}, F\right)$ with $T=B A$. The norm 
$c_{p}$ is given by $c_{p}(T)=\inf \|A\|\|B\|$, where the infimum is over all such factorizations of $T$.

(7) $\left[I_{p, q}, i_{p q}\right], 1 \leqq q \leqq p \leqq \infty$, denotes the ideal of operators factoring through $a$ diagonal $B \in \mathscr{L}\left(L_{p}(\mu), L_{q}(\mu)\right): T \in I_{p, q}(E, F)$ if for some positive measure $\mu$ there are operators $A \in \mathscr{L}(E, L p(\mu)), B \in \mathscr{L}\left(L_{p}(\mu), L_{q}(\mu)\right)$, where $B$ is of the form $B f=f \cdot g$ for some fixed $g \in L_{r}(\mu), 1 / r=1 / q-1 / p$, and $C \in \mathscr{L}\left(L_{q}(\mu), f^{\prime \prime}\right)$, such that $i T=C B A$, where $i$ is the canonical injection of $F$ into $F^{\prime \prime}$. The norm $i_{\mathrm{pq}}$, is given by $i_{\mathrm{pq}}(T)=\inf \|A\|\|B\|\|C\|$.

Observe that $I_{q}(E, F)=I_{\infty, q}(E, F)$ with equality of norms.

(8) $\left[J_{p, q}, j_{p q}\right], 1 \leqq q \leqq p \leqq \infty$, denotes the ideal of operators factoring through $D_{q} \circ \Pi_{p}: T \in J_{p, q}(E, F)$ if $i T$ admits a factorization as follows:

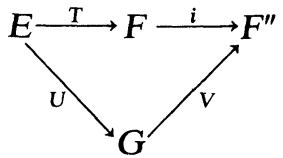

where $U \in \Pi_{p}(E, G)$ and $V \in D_{q}\left(G, F^{\prime \prime}\right)$. Here $j_{p q}(T)=\inf \pi_{p}(U) d_{q}(V)$, the infimum taken over all $U, V, G$.

The last ideal we will discuss is a generalization of an ideal introduced by Kwapien.

(9) $\left[\Gamma_{p, q}, \gamma_{p q}\right]$ is defined as in $\left[I_{p, q}, i_{p q}\right]$, the difference being that $B$ ranges over all members of $\mathscr{L}\left(L_{p}(\mu), L_{q}(\mu)\right)$.

The adjoints, conjugates and duals of these ideals (and several others) are computed in [5.2].

We remark that in the ideals (8) and (9) whenever $p=q$ we will index the ideal and its norm by $p$ alone.

6. Ideal characterizations of $\mathscr{L}_{\mathrm{p}}$-spaces. We first present some characterizations of $\mathscr{L}_{p}$-spaces via Banach ideals. We begin with a characterization of $\mathscr{L}_{\infty}$-spaces.

THEOREM 1 [6.1]. The following assertions are equivalent:

(a) $\Pi_{1}(E, F)=I_{1}(E, F)$ for all $F$; and

(b) $E$ is an $\mathscr{L}_{\infty}$-space.

Using this result Lewis and Stegall [6.2] proved

Theorem 2. Let $E$ be a Banach space. Then $\Pi_{1}(E, F)=N_{1}(E, F)$ for all $F$ if and only if $E^{\prime}$ is isomorphic to $l_{1}(\Gamma)$ for suitable $\Gamma$.

There are some beautiful applications of Theorem 2.

Applications. (1) If $E$ is a complemented subspace of $L_{1}[0,1]$, isomorphic to a subspace of a separable conjugate space, it is isomorphic to $l^{1}$. In particular. (2) $L_{1}[0,1]$ is not isomorphic to a subspace of a separable conjugate space (Gel'fand [6.3], Pelczyński [6.4]. (3) Any separable $\mathscr{L}_{1}$-space which is isomorphic to a conjugate space is isomorphic to $l_{1}$. (We remark that there are many separable $\mathscr{L}_{1}$-spaces. Indeed let $\Phi_{0}: l_{1} \rightarrow L_{1}[0,1]$ be a surjection and let $X_{1}=\Phi_{0}^{-1}(0)$. Let $\Phi_{1}$ be a surjection from $l_{1}$ onto $X_{1}$ and 
$X_{2}=\Phi_{1}^{-1}(0)$. In general let $X_{n}=\Phi_{n-1}^{-1}(0)$. Then all of the $X_{n}$ are separable, nonisomorphic $\mathscr{L}_{1}$-spaces [6.5].)

We now give an omnibus result which includes results of Cohen [6.6], Holub [6.7], Johnson [6.8], Kwapien [6.9], Lewis [6.10] and Persson [6.11], as well as some new results. This result was first proved in [6.12].

THEOREM 3. The following are equivalent $(1 \leqq p \leqq \infty)$ :

(a) $I_{E}$, the identity on $E$, factors through $L_{p}$;

(b) $\Gamma_{p}(F, E) \supseteq C(F, E)$ for all $F$;

(c) $\Gamma_{p^{\prime}}\left(E^{\prime}, F^{\prime}\right) \supseteq C\left(E^{\prime}, F^{\prime}\right)$ for all $F$;

(d) $\Gamma_{p}(E, E) \supseteq C(E, E)$ and $E$ has the metric approximation property;

(e) $\Gamma_{p}^{*}(E, F)=I_{1}(E, F)$ for all $F$;

(f) $\Gamma_{p}^{*}(F, E)=I_{1}(F, E)$ for all $F$;

(g) $\Gamma_{p}^{*}(E, E)=I_{1}(E, E)$ and $E$ has the metric approximation property;

(h) for every Banach space $G$, and every adjoint operator, if $W^{\prime} \in$ $\Pi_{p}\left(E^{\prime}, G^{\prime}\right)$ then $W \in I_{p}(G, E)$;

(i) if $V \in \Pi_{\mathrm{p}^{\prime}}(E, G)$ then $V^{\prime} \in I_{\mathrm{p}^{\prime}}\left(G^{\prime}, E^{\prime}\right)$; and

(j) $\Gamma_{p}(E, F) \supseteq C(E, F)$ for all $F$.

We mention that some other characterizations of $c_{0}, l_{1}, \mathscr{L}_{\infty}$-spaces and $\mathscr{L}_{1}$-spaces are given in [6.13].

It would be of considerable interest to know the situation whenever the range space in Theorem 2 is fixed. We conjecture that if $\Pi_{1}(F, E)=N_{1}(F, E)$ for all Banach space $F$ then $E$ must be finite dimensional.

7. More on $\mathscr{L}_{\mathrm{p}}$-spaces. In [7.1] Grothendieck outlined the theory of tensor products of Banach spaces. This was, in fact, the "beginning" of the theory of ideals of operators on Banach spaces. Indeed, Grothendieck showed the importance of factoring techniques which will be emphasized over and over in this paper.

The crowning achievement of this work of Grothendieck was called by him "the fundamental theorem of tensor products." In terms of matrices this theorem can be stated as follows:

Let $\left(a_{i j}\right)_{i, j=1}^{n}$ be a finite matrix of real numbers such that $\left|\sum_{i, j=1}^{n} a_{i j} t_{i j}\right| \leqq 1$ whenever $\left|t_{i}\right| \leqq 1,\left|s_{j}\right| \leqq 1$. Then, for every set of unit vectors $\left(x_{i}\right)_{i=1}^{n}$ and $\left(y_{j}\right)_{j=1}^{n}$ in a Hilbert space

$$
\left|\sum_{i, j} a_{i j}\left(x_{i}, y_{j}\right)\right| \leqq K_{G},
$$

where $K_{G}$ is an absolute constant. Here $(\cdot, \cdot)$ denotes the inner product in the Hilbert space. We remark that the exact value of $K_{G}$ is not known.

Surprisingly this fundamental paper of Grothendieck lay dormant for many years but was finally taken up again by Lindenstrauss and Pełczyński in 1968 [7.2]. Lindenstrauss and Pełczyński were persuaded to write their paper avoiding the notion of tensor products because "the paper of Grothendieck was quite hard to read and its results were not generally known even to experts in Banach space theory." This remarkable paper of Lindenstrauss and Pełczyński contains the seeds of the application of 
Banach ideals of operators. (Earlier [7.3] Grothendieck essentially used the theory to obtain the Dvoretzky-Rogers theorem.) Before stating a few of their results let us mention that a new proof of the Grothendieck inequality has recently been given by Maurey [7.4]. One of the achievements of the Lindenstrauss-Pełczyński paper was the introduction of the classes of spaces called the $\mathscr{L}_{\mathrm{p}}$-spaces defined in the introduction. The proofs of the theorems below depend heavily on the Grothendieck inequality.

THEOREM 1. Let $X$ be an $\mathscr{L}_{1}$-space and $H$ an $\mathscr{L}_{2}$-space (=isomorph of a Hilbert space). Then $\mathscr{L}(X, H)=\Pi_{1}(X, H)$.

As remarked by Lindenstrauss and Pełczyński it is conceivable that Theorem 1 actually characterizes $\mathscr{L}_{1}$ and $\mathscr{L}_{2}$-spaces. Indeed they obtained the following partial converse to Theorem 1 .

THEOREM 2. Let $X$ and $Y$ be Banach spaces such that $X$ has an unconditional basis and such that $\mathscr{L}(X, Y)=\Pi_{1}(X, Y)$. Then $X$ is isomorphic to $l_{1}$ and $\mathrm{Y}$ is isomorphic to a Hilbert space.

THeOREM 3. Let $X$ be an $\mathscr{L}_{\infty}$-space and $Y$ an $\mathscr{L}_{\mathrm{p}}$-space, $1 \leqq p \leqq 2$. Then $\mathscr{L}(X, Y)=\Pi_{2}(X, Y)$.

Of the numerous applications of these results the following are striking examples.

Application 1. Let $X$ be a complemented subspace of an $\mathscr{L}_{1}\left(\mathscr{L}_{\infty}\right)$-space $Y$ and let $\left(x_{i}\right)$ be a normalized unconditional basis in $X$. Then the basis $\left(x_{i}\right)$ is equivalent to the unit vector basis $\left(e_{i}\right)$ in $l_{1}\left(c_{0}\right)$, i.e. the operator $T$ defined by $T x_{i}=e_{i}$ is an isomorphism.

For the next application we consider the complex Banach space $L_{1}(\mu)$, where $\mu$ is Haar measure on $\{z:|z|=1\}$. Let $H_{1}$ be the closure of the polynomials $\sum_{k=0}^{n} a_{k} z^{k}$ in $L_{1}(\mu)$.

Proposition. $\mathscr{L}\left(H_{1}, l_{2}\right) \neq \Pi_{1}\left(H_{1}, l_{2}\right)$.

We thus obtain the classic result of D. J. Newman (see [7.5, p. 154]).

APPLiCATION 2. Every isomorphic image of $H_{1}$ in an arbitrary $\mathscr{L}_{1}$-space $X$ is uncomplemented.

Another application is Grothendieck's characterization of Hilbert spaces.

Application 3. A Banach space $X$ is isomorphic to a Hilbert space if and only if it is isomorphic to a subspace of an $\mathscr{L}_{1}$-space and to a quotient of an $\mathscr{L}_{\infty}$-space.

Of course, if the roles of the $\mathscr{L}_{1}$ - and $\mathscr{L}_{\infty}$-spaces are interchanged, every Banach space meets the requirement.

Finally if $\mathscr{L}(X, Y)=\Pi_{1}(X, Y)$ there is a bit one can say about $X$. More precisely,

Proposition. If $\mathscr{L}(X, Y)=\Pi_{1}(X, Y)$ then

$1^{\circ} \mathscr{L}\left(X, l_{2}\right)=\Pi_{1}\left(X, l_{2}\right)$;

$2^{\circ}$ if $\sum_{n=1}^{\infty} x_{n}$ is unconditionally convergent in $X$ then $\sum_{n=1}^{\infty}\left\|x_{n}\right\|^{2}<+\infty$; and,

$3^{\circ}$ if $Z$ is any $\mathscr{L}_{\infty}$-space, $\mathscr{L}(Z, X)=\Pi_{2}(Z, X)$.

For a detailed study of this proposition, see [7.6]. 
8. Schwartz duality theorem. Following Kwapien [8.1] we present the Schwartz duality theorem without the theory of cylindrical measures and radonifying operators.

We first need to extend the notion of $p$-absolutely summing operator to include the interval $[0,1)$. For $0<p<+\infty$ define a $p$-absolutely summing operator in the obvious way, i.e., $T$ is $p$-absolutely summing if there is a $C$ such that for $x_{1} \cdots x_{n} \in E$

$$
\sum_{i=1}^{n}\left\|T x_{i}\right\|^{p} \leqq C \sup _{\|f\|=1} \sum_{i=1}^{n}\left|\left\langle x_{i}, f\right\rangle\right|^{p} .
$$

For $p=0$ we say that $T$ is 0 -absolutely summing (and write $T \in \Pi_{0}(E, F)$, if for each $\varepsilon>0$ there is a $\delta>0$ such that for $x_{1} \cdots x_{n} \in E$ and

$$
\sup _{\|f\|=1} \sum_{i=1}^{n} \frac{1}{n} \min \left[1,\left|\left\langle x_{i}, f\right\rangle\right|\right]<\delta
$$

it follows that $\sum_{i=1}^{n} n^{-1} \min \left[1,\left\|T x_{i}\right\|\right]<\varepsilon$. This last definition is a reformulation of Schwartz's definition of a radonifying operator.

Now let $\mu$ be a probability measure on a Hausdorff space $\Omega$ and $0 \leqq p \leqq \infty$. A linear operator $\nu: E \rightarrow L_{p}(\Omega, \mu)$ is $p$-decomposable if there is a $\varphi: \Omega \rightarrow E^{\prime}$ such that

(a) for each $x \in E,\langle x, \varphi(\cdot)\rangle$ is $\mu$-measurable and equal to $\nu(x) \mu$-a.e.; and

(b) there is $f \in L_{p}(\Omega, \mu)$ such that $\|\varphi(\omega)\| \leqq f(\omega) \mu$-a.e.

THEOREM (THE SCHWARTZ DUALITY THEOREM). Let $E$ be a Banach space and $0 \leqq p<+\infty$. If $\nu: E \rightarrow L_{p}(\Omega, \mu)$ is $p$-decomposable then $\nu$ is $p$-absolutely summing.

For $0 \leqq q<p<2$ there is an isomorphic embedding of $L_{p}[0,1]$ into $L_{q}(\Omega, \mu)$. If $p=2$ the same is true for all $0 \leqq q<+\infty$. We denote such an isomorphism by $\nu_{p}$.

THEOREM 2. Let $1<p \leqq \infty$ and $T: L_{p}[0,1] \rightarrow L_{2}[0,1]$. The following are equivalent:

(a) $\nu_{2} T$ is $q$-decomposable for all $q<\infty$;

(b) $\nu_{2} T$ is 0-decomposable;

(c) $T$ is 0 -absolutely summing; and,

(d) $T$ is $p^{\prime}$-absolutely summing.

TheOREM 3. Let either $1 \leqq p<2$ and $0 \leqq q<p$ or $p=2$ and $0 \leqq q<+\infty$. Let $F$ be an $\mathscr{L}_{p}$-space. If $T \in \mathscr{L}(E, F)$ and $T^{\prime} \in \Pi_{q}\left(F^{\prime}, E^{\prime}\right)$ then $T \in \Pi_{0}(E, F)$.

Application 1. Let $F$ be an $\mathscr{L}_{r}$-space where $1<r \leqq 2$.

(a) If $0 \leqq p \leqq 2$ then $\Pi_{p}(F, E)=\Pi_{0}(F, E)$;

(b) If $2 \leqq p<\infty$ then $\Pi_{\mathrm{p}}(E, F)=\Pi_{2}(E, F)$.

In particular for an isomorph of a Hilbert space (i.e., an $\mathscr{L}_{2}$-space), $H$, an operator is $p$-absolutely summing for some $p, 0 \leqq p<\infty$ if and only if it is 2-absolutely summing (=Hilbert-Schmidt). 
Application 2. Let $F$ be an $\mathscr{L}_{r}$-space, $1<r<\infty$. Then $\mathrm{Il}_{1}(F, E)=\mathrm{II}_{0}(F, E)$ for any Banach space $E$.

Application 3. Let $2<p<\infty$. Let $E$ be an $\mathscr{L}_{\infty}$-space and $F$ an $\mathscr{L}_{p}$-space. Then

(a) $\mathscr{L}(E, F)=\Pi_{q}(E, F)$ for $q>p$; but,

(b) $\mathscr{L}(E, F) \neq \Pi_{p}(E, F)$.

(We mention that Saphar [8.2], [8.3] has obtained (a) of Application 3 replacing $\Pi_{q}$ with $I_{q}$.)

Application 4 . Let $F$ be a subspace of an $\mathscr{L}_{1}$-space. Then

(a) $\Pi_{1}(F, E)=\Pi_{2}(F, E)$; and,

(b) if $p \geqq 2, \Pi_{p}(E, F)=\Pi_{2}(E, F)$ for any Banach space $E$.

Many authors have considered the question: "When is the adjoint of an operator $p$-absolutely summing?" The most satisfying answer, we feel, was given by Cohen [8.4] since it retains the series interpretations of these operators. Stated in the language of ideals Cohen's result is $\Pi_{p}^{\prime}=D_{p^{\prime}}$ and $D_{p}^{\prime}=\Pi_{p^{\prime}}$.

Aside from the work of Kwapien here and the fundamental result of Schwartz we mention the papers of Garling [8.5] and Nielsen [8.6].

9. Banach spaces $X$ for which $\Pi_{2}\left(\mathscr{L}_{\infty}, X\right)=\mathscr{L}\left(\mathscr{L}_{\infty}, X\right)$. The GrothendieckLindenstrauss-Pełczyński theorem and the previous results of Kwapien indicate that perhaps a space $X$ for which $\mathscr{L}(E, X)=\Pi_{2}(E, X)$ for an $\mathscr{L}_{\infty^{-}}$ space $E$ is necessarily a subspace of some $L_{1}(\mu)$. This problem was considered by Dubinsky, Pełczyński and Rosenthal [9.1]. They show that the above conjecture is false. Moreover they give a complete characterization of such spaces whenever $X$ has an unconditional basis. First we remark that if $\mathscr{L}(E, X)=\Pi_{2}(E, X)$ for some $\mathscr{L}_{\infty}$-space $E$ then the equality holds for all $\mathscr{L}_{\infty}$-spaces; thus, the notation $\mathscr{L}\left(\mathscr{L}_{\infty}, X\right)=\Pi_{2}\left(\mathscr{L}_{\infty}, X\right)$.

EXAMPLE 1. Let $X=\left(\bigoplus E_{n}\right)_{l_{2}}$ where each $E_{n}=l_{1}$.

(a) $\Pi_{2}\left(\mathscr{L}_{\infty}, X\right)=\mathscr{L}\left(\mathscr{L}_{\infty}, X\right)$, and

(b) $X$ is not isomorphic to any subspace of an $\mathscr{L}_{1}$-space.

Actually one can obtain a reflexive space with properties (a) and (b) of Example 1. Indeed let $X=\left(\bigoplus E_{n}\right)_{l_{2}}$ where $E_{n}=l_{1}^{n}$.

Although there are no known necessary and sufficient conditions on a general space $X$ guaranteeing $\Pi_{2}\left(\mathscr{L}_{\infty}, X\right)=\mathscr{L}\left(\mathscr{L}_{\infty}, X\right)$, there are rather simple conditions guaranteeing this property for $X$ in the class of spaces having unconditional bases.

THEOREM 1. Let $X$ have an unconditional basis $\left(u_{n}\right)$. Then $\mathscr{L}\left(\mathscr{L}_{\infty}, X\right)=\Pi_{2}\left(\mathscr{L}_{\infty}, X\right)$ if and only if every operator from $c_{0}$ into $X$, which takes the nth unit vector of $c_{0}$ into a multiple of $u_{n}$ for each $n$ (i.e., a diagonal mapping), is 2-absolutely summing.

It is obvious that if $\mathscr{L}\left(\mathscr{L}_{\infty}, X\right)=\Pi_{2}\left(\mathscr{L}_{\infty}, X\right)$ the same is true for any subspace of $X$. However, it is easily seen that the property is not preserved by quotients.

The following result gives a property which preserves the above equality for quotients. 
Theorem 2. Let $X$ be a Banach space. Suppose that there exists a probability space $(\Omega, \Sigma, \mu)$ and a sequence of functions $\left(f_{n}\right)$ in $L_{1}(\Omega, \Sigma, \mu)$, and a constant $C>0$ such that

$$
C^{-1}\left(\sum_{i=1}^{m}\left|a_{i}\right|^{2}\right)^{1 / 2} \leqq \int_{\Omega}\left|\sum_{i=1}^{m} a_{i} f_{i}(\omega)\right| \mu d(\omega)
$$

for any scalars $a_{1}, a_{2}, \cdots, a_{m}(m=1,2, \cdots)$, and

$$
\int_{\Omega}\left\|\sum_{i=1}^{m} x_{i}^{*} f_{i}(\omega)\right\| \mu d(\omega) \leqq C\left(\sum_{i=1}^{m}\left\|x_{i}^{*}\right\|^{2}\right)^{1 / 2}
$$

for any $x_{1}^{*}, \cdots, x_{m}^{*}$ in $X^{*}$.

Then $\Pi_{2}\left(\mathscr{L}_{\infty}, X\right)=\mathscr{L}\left(\mathscr{L}_{\infty}, x\right)$. Moreover, every quotient space $Y$ of $X$ has the same property (indeed the same $\left(f_{n}\right)$ and $C$ suffice). Consequently $\Pi_{2}\left(\mathscr{L}_{\infty}, Y\right)$ $=\mathscr{L}\left(\mathscr{L}_{\infty}, Y\right)$ for all such $\mathrm{Y}$.

Recall that a Banach space $X$ is a $\mathscr{D}_{1}$-space if there is some $K>1$ and a sequence of subspaces $X_{n} \subset X$ such that $d\left(X_{n}, l_{1}^{n}\right) \leqq K$ for $n=1,2, \cdots$.

THEOREM 3. If $X$ is a Banach space such that $\Pi_{2}\left(\mathscr{L}_{\infty}, Y\right)=\mathscr{L}\left(\mathscr{L}_{\infty}, Y\right)$ for every quotient space $Y$ of $X$, then $X$ is not a $\mathscr{D}_{1}$-space.

Say that a Banach space $X$ has the Orlicz property if for each unconditionally convergent series $\sum_{m=1}^{\infty} x_{m}$ in $X, \sum_{m=1}^{\infty}\left\|x_{m}\right\|^{2}<+\infty$. (Orlicz [9.2] discovered that for $1 \leqq p \leqq 2$, the $L_{p}(\mu)$-spaces have this property.)

Application. If $\Pi_{2}\left(\mathscr{L}_{\infty}, X\right)=\mathscr{L}\left(\mathscr{L}_{\infty}, X\right)$ then $X$ has the Orlicz property.

It is not known if the converse is true.

10. Subspaces of $L_{p}$. In this section we consider a fundamental paper of H. Rosenthal [10.1], which in turn was motivated by a paper of J. Bretagnelle and D. Dachunha-Castelle [10.2]. Rosenthal's work is a beautiful application of the theory of $p$-absolutely summing operators.

We begin with a characterization of the $\pi_{\mathrm{p}}$-norm of operators on $l_{\infty}^{n}$. In what follows, $\left\{e_{1} \cdots e_{n}\right\}$ will denote the natural basis of $l_{\infty}^{n}$.

Lemma 1. Let $X$ be a Banach space, $n$ a positive integer; $K>0,1<q<\infty$ and $1 / q+1 / p=1$. Let $T \in \mathscr{L}\left(l_{\infty}^{n}, X\right)$ with $T e_{i}=x_{i}$. The following are equivalent:

(1) $\pi_{q}(T) \leqq K$;

(2) for all integers $m$ and matrices $\left(y_{i j}\right)(1 \leqq j \leqq n, 1 \leqq i \leqq m)$ of scalars we have

$$
\left(\sum_{i=1}^{m}\left\|\sum_{j=1}^{n} y_{i j} x_{i}\right\|^{q}\right)^{1 / q} \leqq K \sup _{1 \leq j \leq n}\left(\sum_{i=1}^{m}\left|y_{i j}\right|^{q}\right)^{1 / q} .
$$

Using Lemma 1 it is easy to prove the next result.

Lemma 2. Let $1<q<\infty, 1 / p+1 / q=1, X$ a Banach space and $K>0$. The following are equivalent:

(1) for any compact Hausdorff space $S$

$$
\mathscr{L}(C(S), X)=\Pi_{q}(C(S), X)
$$


and

$$
\pi_{q}(T) \leqq K\|T\| \quad \text { for any } T \in \mathscr{L}(C(S), X) ;
$$

(2) for each $n, x_{1} \cdots x_{n} \in X$ and matrices $\left(y_{i j}\right)(1 \leqq i \leqq n, 1 \leqq j \leqq n)$ of scalars,

$$
\left(\sum_{i}\left\|\sum_{j} y_{i j} x_{i}\right\|^{q}\right)^{1 / q} \leqq K \sup _{j}\left(\sum_{i}\left|y_{i j}\right|^{2}\right)^{1 / q} \sup _{\|f\|=1} \sum_{i}\left|\left\langle x_{i}, f\right\rangle\right| ;
$$

(3) if $B$ is any Banach space and $T \in N_{p}(X, B)$ with $\nu_{p}(T) \leqq 1$ then $T \in$ $\Pi_{1}(X, B)$ and $\pi_{1}(T) \leqq 1$.

Our first theorem is the principal tool for the main results.

THeOReM 1. Let $R$ be a subspace of $L_{1}(\mu), 1<p<+\infty, 1 / p+1 / q=1$, $K<+\infty$. The following are equivalent:

(1) for any positive integer $n$ and elements $r_{1} \cdots r_{n}$ of $R$,

$$
\int\left(\sum\left|r_{i}\right|^{p}(t)\right)^{1 / p} d \mu(t) \leqq K\left(\sum\left\|r_{i}\right\|^{p}\right)^{1 / p}
$$

(2) if $J: L^{\infty}(\mu)$ onto $R^{*}$ is the natural map (i.e., the adjoint of the inclusion map $\left.R \hookrightarrow L_{1}(\mu)\right)$, then $\pi_{q}(J) \leqq K$; and

(3) there is a nonnegative measurable function $\phi$ with $\int \phi d \mu \leqq 1$, so that for all $r \in R, r(t)=0$ for almost all $t \in\{t: \phi(t)=0\}$ and such that

$$
\left(\int|r|^{p}(t) \phi^{1-p}(t) d \mu(t)\right)^{1 / p} \leqq K \int|r(t)| d \mu(t)
$$

Moreover if $1<p \leqq 2$ and any of (1), (2), or (3) hold, then

$$
\mathscr{L}\left(\mathrm{C}(\mathrm{S}), \mathrm{R}^{*}\right)=\Pi_{q}\left(\mathrm{C}(\mathrm{S}), \mathrm{R}^{*}\right)
$$

and if $T \in \mathscr{L}\left(C(S), R^{*}\right), \Pi_{q}(T) \leqq K\|T\|$.

Using Theorem 1 we can now define a characteristic $I_{p}(R)$ for $R$ a subspace of $L_{1}(\mu)$ and $1<p<\infty$. Indeed let

$$
\begin{array}{rlrl}
\mathbf{I}_{p}(R) & =\pi_{q}(J) & & \text { if } J \text { is } q \text {-absolutely summing, } \\
& =+\infty & \text { if } J \text { is not } q \text {-absolutely summing. }
\end{array}
$$

Here, again, $J$ is the natural mapping from $L^{\infty}(\mu)$ onto $R^{*}$.

Applications AND FACTs. Let $R$ be a subspace of $L_{1}(\mu)$ and suppose $1<p \leqq p^{\prime}<\infty$. Then:

(a) $I_{p}(R) \leqq I_{p^{\prime}}(R)$.

(b) If $R$ is an $\mathscr{L}_{2}$-space then $I_{2}(R)<+\infty$; moreover, if $I_{p}(R)<+\infty$ for some $p \geqq 2$, then $R$ is an $\mathscr{L}_{2}$-space.

(c) If $\tilde{R}$ and $R$ are isomorphic subspaces of $L_{1}(\mu)$ then $I_{p}(\tilde{R}) \leqq d(R, \tilde{R}) I_{p}(R)$.

(d) $I_{p}\left(l^{p}\right)=+\infty$ for all $1<p<2$. (This last result was observed by S. Kwapien.)

(e) Given any subinterval $A$ of $[2, \infty)$ containing 2 , there is an infinitedimensional subspace $R$ of $L_{1}[0,1]$ such that $A=\left\{p \geqq 2: I_{p}(R)<+\infty\right\}$.

(f) Given any subinterval $B$ of $[1,2]$ containing 1 , there is a subspace $R$ of $L_{1}[0,1]$ such that $B=\left\{p \leqq 2: R\right.$ imbeds in $\left.L_{p}\right\}$.

In particular, (e) shows that for $\mathscr{L}_{2}$-subspaces of $L_{1}[0,1]$, whether or not 
$I_{p}(R)<+\infty$ for $p>2$ depends on the manner in which $R$ is imbedded in $L_{1}[0,1]$.

THEOREM 2. Let $1<p \leqq 2,1 / p+1 / q=1$, and $R$ a subspace of $L^{p}(\nu)$. Then either

(i) there is a $p^{\prime}>p$ such that $R$ imbeds in $L_{p^{\prime}}(\nu)$; or

(ii) for any $\varepsilon>0$ there is a subspace $Y$ of $R$, an invertible operator $T$ from $Y$ onto $l_{p}$, and a projection $P$ from $L^{p}(\nu)$ onto $Y$ such that $\|T\|\left\|T^{-1}\right\| \leqq$ $1+\varepsilon$ and $\|P\| \leqq 1+\varepsilon$;

(iii) reflexive quotients of $C(S)$-spaces are isomorphic to quotients of $L_{p}(\mu)$ spaces for some $p<+\infty$. In particular (Grothendieck) complemented reflexive subspaces of $C(S)$-spaces are finite dimensional; and

(iv) every subspace of $L_{1}[0,1]$ contains a subspace with an unconditional basis.

Our next result is analogous to Theorem 2 .

Theorem 3. Let $1<p<2$, and $X$ a subspace of $L_{p}(\mu)$. The following are equivalent:

(1) $I_{p}(X)<\infty$;

(2) X contains no subspace isomorphic to $l_{p}$;

(3) $X$ imbeds in $L_{p^{\prime}}(\mu)$ for some $p^{\prime}>p$;

(4) $\mathscr{L}\left(X, l_{p}\right)=K\left(X, l_{p}\right)$; and

(5) $\mathscr{L}\left(C(S), X^{*}\right)=\Pi_{q}\left(C(S), X^{*}\right)$ where $1 / p+1 / q=1$.

Maurey [10.3] has generalized some of the above results as follows: for a Banach space $E$ let $I_{E}=\left\{q>0 \mid\right.$ for any $F, \Pi_{q}(E, F)=\Pi_{p}(E, F)$ for some $p \in(0,1)\}$.

'THEOREM 4. For a Banach space $E$ and $q \in[1,2)$ the following are equivalent:

(a) $q \notin I_{E}$;

(b) for all $\varepsilon>0$ and for all $n$ there are operators $U, V$ with $U \in \mathscr{L}\left(l_{q^{\prime}}^{n}, E\right)$, $V \in \mathscr{L}\left(E, l_{\infty}^{n}\right)$ such that $\|U\| \leqq 1+\varepsilon,\|V\|=1$ and $V \circ U$ is the inclusion map.

As a special case we obtain

Corollary 1. For a Banach space $E$ the following are equivalent:

(a) $1 \notin I_{\mathrm{E}}$; and,

(b) for each $\varepsilon>0 E$ is an $\mathscr{S}_{\infty, 1+\varepsilon}$-space.

Application. If $E$ is a Banach space and if there is a $q \geqq 1$ such that for any summable sequence $\left(x_{n}\right)$ in $E, \sum\left\|x_{i}\right\|^{q^{\prime}}<+\infty$ then $(0, q) \subset I_{E}$.

The converse of this statement is false, e.g. if $q=2$ then for $1 \leqq p<2, L_{p^{\prime}}(\mu)$ provides a counterexample.

Corollary 2. For any Banach space $E, I_{E}$ is an open segment or is $(0,2]$.

The case $(0,2)$ can occur, e.g. the Orlicz space $L^{\phi}([0,1], d t)$ where $\phi(t)=t^{2} \log (t+1)$. (See [10.4] for the appropriate definitions.) 
THEOREM 5. If $E$ is a Banach space and $p$, $q$ satisfy $0<p<q$ and if for any $F, \Pi_{q}(E, F)=\Pi_{p}(E, F)$ then $q \in I_{E}$.

We will continue with results on $I_{E}$ in the next section.

Recently Nicole Tomczak has communicated still another application of the Rosenthal-Maurey work.

THEOREM 6. If $1<p<2$ and $E$ and $F$ are Banach spaces such that $\mathscr{L}(E, F)=\Pi_{p}(E, F)$, i.e., $\langle E, F\rangle$ is p-trivial, then $\mathscr{L}(E, F)=\Pi_{0}(E, F)$.

This answers a problem raised in [10.5],

11. The weakly nuclear norm and LUST. In studying the equality $\mathscr{L}\left(\mathscr{L}_{\infty}, X\right)=\Pi_{2}\left(\mathscr{L}_{\infty}, X\right)$ Dubinsky, Pełczyński and Rosenthal introduced the important notion of local unconditional structure (LUST). This concept is a generalization of the notion of an unconditional basis and has proved quite useful recently in gaining insight into the structure of certain Banach spaces.

First, suppose $X$ is a Banach space with an unconditional basis. The unconditional basis constant of $X$ is defined by

$$
\chi(X)=\inf _{\left\{e_{i}\right\}} \sup _{\varepsilon_{i}= \pm 1, x_{i}}\left\|\sum_{i} \varepsilon_{i} x_{i} e_{i}\right\| /\left\|\sum_{i} x_{i} e_{i}\right\|
$$

where the supremum is taken over all choices of signs $\varepsilon_{i}= \pm 1$ with $\varepsilon_{i}=1$ for all but finitely many $i$, and over all vectors $\sum_{i} x_{i} e_{i}$ in $E$, and the infimum is over all possible unconditional bases $\left\{e_{i}\right\}$ of $E$.

A Banach space $E$ has LUST if there is a family $\left\{E_{i}\right\}$ of finite dimensional spaces, each with unconditional basis constant 1 , and a $C \geqq 1$ such that for each finite dimensional subspace $F \subset E$ there is an $i$ and an operator $T: E_{i} \rightarrow E$ such that $T\left(E_{i}\right) \supset F$ and $\|e\| \leqq\|T e\| \leqq C\|e\|$ for $e \in E_{i}$, and moreover, for each $i$ there is an operator $S_{i}: E_{i} \rightarrow E$ with $\|e\| \leqq\left\|S_{i} e\right\| \leqq C\|e\|$ for $e \in E_{i}$.

Following Gordon and Lewis [11.1] we give a definition closely related to LUST, in terms of a certain Banach ideal.

An operator $U: E \rightarrow F$ is weakly nuclear if $U=\sum_{n=1}^{\infty} f_{n} \otimes y_{n}$ and the series is unconditionally convergent in the operator norm. The weakly nuclear norm $\eta$ is defined by

$$
\eta(U)=\inf \varepsilon_{1}\left(f_{n} \otimes y_{n}\right)
$$

where the infimum is over all possible representations of $U$.

LEMMA. For any operator $U, \eta(U)=\inf \|\alpha\| \chi(X)\|\beta\|$ where the infimum is over all factorizations

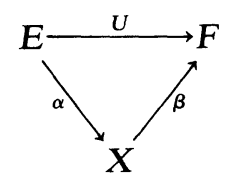

where $\alpha \in K(E, X), \beta \in \mathscr{L}(X, F)$ and $X$ has an unconditional basis.

From the lemma, it is clear that if $\operatorname{dim} E=n$ then $\eta\left(\mathrm{id}_{E}\right) \leqq \chi(E)$.

Definition. Let $E$ be a Banach space. If $\sup \left\{\eta\left(j_{F}\right): F \subset E\right.$, $\operatorname{dim} F<+\infty\}<+\infty$, we denote this constant by $\chi_{u}(E)$. Here $j_{F}$ denotes the inclusion mapping $F \hookrightarrow E$. 
FACTS. (a) $\chi_{u}(E) \leqq \chi(E)$;

(b) if $\operatorname{dim} E<+\infty, \chi_{u}(E)=\eta\left(\mathrm{id}_{E}\right)$;

(c) if $\chi_{u}(E)=+\infty$ then $E$ is not complemented in a space with an unconditional basis;

(d) if $F$ is a finite dimensional subspace of $E$ and $P: E \rightarrow F$ a projection, then $\chi_{u}(E)\|P\| \geqq \chi_{u}(F)$. Clearly if $E$ has LUST then $\chi_{u}(E)<+\infty$.

That LUST is a true generalization of the notion of an unconditional basis is immediate from the fact that any $C(K)$-space has LUST but, e.g., $C[0,1]$ does not have an unconditional basis.

Before considering properties of spaces with LUST, we first give a fundamental result of Gordon and Lewis [11.1] which shows that not every Banach space has LUST.

THEOREM 1. $\eta\left(\mathscr{L}\left(l_{2}^{n}, l_{2}^{n}\right)\right) \geqq(2 / 3 \pi) \sqrt{ }$. In particular $\chi\left(\mathscr{L}\left(l_{2}^{n}, l_{2}^{n}\right)\right) \geqq K n^{1 / 2}$, and since $\mathscr{L}\left(l_{2}^{n}, l_{2}^{n}\right)$ is complemented in $K\left(l_{2}, l_{2}\right), K\left(l_{2}, l_{2}\right)$ does not have LUST.

We should remark that the sequence $\left\{\mathscr{L}\left(l_{2}^{n}, l_{2}^{n}\right)\right\}$ was the first sequence of finite dimensional Banach spaces $\left\{E_{n}\right\}$ shown to have the property that $\chi\left(E_{n}\right) \rightarrow \infty$.

There are numerous applications of the theorem. Indeed, this result settled many outstanding problems. We mention only two applications here. Others are given in $\$ 12$.

Applications. (1) $\sigma_{p}(H)$ does not have LUST if $p \neq 2 ;(2)$ there is a compact, nonweakly nuclear operator on $K\left(l_{2}, l_{2}\right)$ (problem of Pietsch).

The next result motivates the remainder of this section.

Theorem 2. If $T \in \Pi_{1}(E, F)$ then $\nu_{1}(T) \leqq \pi_{1}(T) \chi_{u}(E)$. Thus, if $E$ has LUST, $\Pi_{1}(E, \cdot) \subset \Gamma_{1}(E, \cdot)$.

We now define the class GL. Roughly speaking, a Banach space $X$ is in the class GL if absolutely summing operators from $X$ factor locally through $l_{1}$. More precisely we make the following definition.

Definition. A Banach space $X$ is in the class $G L$ if and only if for any $U \in \Pi_{1}(X, Y)$ and for any finite dimensional subspace $X_{1}$, of $X$ there are operators $\alpha: X_{1} \rightarrow l_{1}^{n}, \beta: l_{1}^{n} \rightarrow Y,\|\alpha\|=1,\|\beta\| \leqq \pi_{1}(U)$ such that $\beta \alpha=\left.U\right|_{X_{1}}$.

THEOREM 3. If $Y$ is a conjugate space and $X \in G L$ then $\Pi_{1}(X, Y) \subset \Gamma_{1}(X, Y)$.

Let us first list some spaces belonging to the class GL:

(a) quotients of $C(K)$-spaces and subspaces of $L_{1}$-spaces,

(b) all Banach lattices,

(c) any space with LUST.

Aside from the spaces mentioned earlier which are not in the class GL, we remark that recently Pełczyński [11.2] has shown that there are "classical" Banach spaces which are not in the class GL.

Theorem 4. The disk algebra $A(D)$, its dual space $A^{*}$, and $H^{\infty}$ are not in the class GL. 
Here is an interesting application of this result:

Application. Let $n \geqq 2$ and let $\Omega$ be a domain in $C^{n}$. Then $A(D)$ is not isomorphic to $A(\Omega)$.

Recently, D. Lewis [11.3] has obtained the following result which shows the complete picture:

TheOREM 5. Let $[A, \alpha]$ be a Banach ideal such that $A\left(l_{2}, l_{2}\right)$ is not equivalent to the Hilbert-Schmidt operators. Then $\left[A\left(l_{2}, l_{2}\right), \alpha\right]$ does not have LUST. Indeed, such an ideal is not in the class GL.

We now discuss two papers, one by W. B. Johnson [11.4] and the other by Johnson, T. Figiel and L. Tzafriri [11.5].

We say that $Y$ is finitely representable in $X$ provided that for each $\varepsilon>0$ and each finite dimensional subspace $E$ of $Y$, there is a subspace $F$ of $X$ for which $d(E, F) \leqq 1+\varepsilon$; and $X$ is superreflexive [11.6], [11.7] if any Banach space which is finitely representable in $X$ is reflexive. It is now known that $X$ is superreflexive if and only if $X$ admits an equivalent uniformly convex norm [11.8].

Our next result is a structural theorem for spaces with LUST.

THEOREM 6. Suppose X has LUST. Then either

(1) $X$ is superreflexive, or

(2) $X \supset E_{n}$ with $d\left(E_{n}, l_{\infty}^{n}\right) \rightarrow 1$, or

(3) $X \supset E_{n}$ with $d\left(E_{n}, l_{1}^{n}\right) \rightarrow 1$ and $E_{n}$ is $(1+1 / n)$-complemented in $X$.

To again emphasize the relationships between LUST and unconditional bases we mention a result of Tzafriri [11.9]: If $X$ has an unconditional basis then $X$ is an $\mathscr{S}_{\mathrm{p}}$-space for some $p=1,2$, or $\infty$.

There is an application of Theorem 6 to ideals.

Application. Let $X$ and $Y$ be Banach spaces and suppose that $X$ is a subspace of a space with LUST which contains no sequence $\left(E_{n}\right)$ with $d\left(E_{n}, l_{\infty}^{n}\right) \rightarrow 1$. Then

$$
K(X, Y) \neq N(X, Y) \text { and } K(Y, X) \neq N_{1}(Y, X) .
$$

It is possible to characterize LUST using Banach lattices.

THEorem 7. A Banach space $X$ has LUST if and only if there is a Banach lattice $L, a \lambda<+\infty$, and a subspace $Y$ of $L, d(Y, X)<+\infty$ such that for each finite dimensional subspace $E$ of $L$ there is an operator $T=T_{E}: E \rightarrow Y$ for which $\left.T\right|_{E \cap Y}=\mathrm{id}_{\mathrm{E} \cap \mathrm{Y}}$ and $\|T\|\left\|T^{-1}\right\| \leqq \lambda$.

Thus,

Corollary 1. A Banach space $X$ has $\chi_{u}(X)<+\infty$ if and only if $X^{* *}$ is complemented in a Banach lattice. Also $X$ has LUST if and only if $X^{* *}$ has LUST.

COROllary 2. If $X$ is complemented in a Banach lattice $L$ and $X$ is $a$ $\mathscr{D}_{\infty}$-space $\left(=\mathscr{Y}_{\infty}\right.$-space) then $X$ has LUST.

We next recall that every subspace of $L_{1}[0,1]$ contains a subspace with an 
unconditional basis. The next result shows that this remains true for certain Banach lattices. (See [11.10] for appropriate definitions.)

THEOREM 8. Let $L$ be a $\sigma$-complete and $\sigma$-order continuous Banach lattice. Then every subspace of L contains a subspace with an unconditional basis.

Corollary 3. If $X$ has LUST and $X$ is not a $\mathscr{D}_{\infty}$-space, then every subspace of $X$ contains a subspace with an unconditional basis.

Finally, from [11.11] we obtain the following striking result:

THEOREM 9. If $X$ is a subspace of a Banach lattice which is not $a$ $\mathscr{D}_{\infty}$-space, then $X$ contains uniformly complemented $E_{n}$ with $\sup d\left(E_{n}, l_{1}^{n}\right)<+\infty$ or $\sup d\left(E_{n}, l_{2}^{n}\right)<+\infty$.

Thus if $X$ has LUST, $X$ is either an $\mathscr{Y}_{1^{-}}, \mathscr{S}_{2^{-}}$or $\mathscr{S}_{\infty}$-space.

It is conjectured that any Banach space is an $\mathscr{S}_{1^{-}}, \mathscr{S}_{2^{-}}$or $\mathscr{S}_{\infty}$-space!

12. Parameters. In studying the geometry of Banach spaces, numerous parameters have been introduced. Perhaps the "projection constant" is the best known and most often studied among these parameters. Ideal norms provide a unified approach for attaching parameters to a Banach space. Indeed, if $[A, \alpha]$ is a Banach ideal and $E$ a Banach space, define $\alpha(E)=\alpha\left(\mathrm{id}_{\mathrm{E}}\right)$.

Some of the most beautiful work done in recent years, utilizing ideal parameters to the fullest, has been done by Y. Gordon [12.1], [12.2], Gordon and Garling [12.3] and the superb papers of Gordon and Lewis [12.4], [12.5]. See also the related papers [12.6] of A. Pietsch.

In this section we give a few of the numerous results.

We first recall some definitions of asymmetry. For a study of the following concepts see, in addition to the papers above, [12.7].

Let $E$ be a Banach space and $G$ a multiplicative group of operators in $\mathscr{L}(E, E)$ and let $G^{\prime}=\{t \in \mathscr{L}(E, E): \operatorname{tg}=g t, \quad g \in G\}, G_{E}=\{J \in \mathscr{L}(E, E): J$ an isometry\}. We say $E$ has enough symmetries if $G_{E}^{\prime}$ consists only of the scalar multiples of $\mathrm{id}_{\mathrm{E}}$.

The asymmetry constant $S(E)$ is defined to be the infimum of $\lambda>0$ for which there is a group $G \subset \mathscr{L}(E, E)$ of invertible, onto operators such that

$$
\sup \{\|g\|: g \in G\} \leqq \lambda \text { and } G^{\prime}=\left\{\alpha \mathrm{id}_{\mathrm{E}}\right\} .
$$

It can be shown that, in fact,

$$
S(E)=\inf \{d(E, F) ; F \text { has enough symmetries }\} \text {. }
$$

Theorem 1. If $\operatorname{dim} E=n$ and $[A, \alpha]$ is any Banach ideal then

$$
n \leqq \alpha(E) \alpha^{\Delta}(E) \leqq n(S(E))^{2} \text {. }
$$

In particular if $E$ has enough symmetries $\alpha(E) \alpha^{\Delta}(E)=n$.

Theorem 1 was generalized in [12.8]. Without going into technical details we state a special case of this generalization. 
Application. For each injection map $I: l_{p}^{n} \rightarrow l_{q}^{n}, \alpha^{\Delta}(I) \alpha\left(I^{-1}\right)=n$. Here $p$, $q$ are arbitrary and $\alpha$ is any ideal norm.

If $E$ has a basis $B=\left\{e_{i}\right\}$, and $\sigma$ is a finite permutation of the integers, define $\sigma \in \mathscr{L}(E, E)$ by $\sigma\left(e_{i}\right)=e_{\sigma(i)}$. The diagonal asymmetry constant $\delta(B)$ is defined as $\sup _{\sigma}\|\sigma\|$ and the diagonal asymmetry constant of $E, \delta(E)$, is defined as $\inf \{\delta(B): B$ a basis for $E\}$.

If $\varepsilon=\left(\varepsilon_{i}\right)$ is a sequence of \pm 1 , with $\varepsilon_{i}=1$ except for a finite number of $i$ then $g_{\varepsilon} \in \mathscr{L}(E, E)$ is defined by $g_{\varepsilon}\left(e_{i}\right)=\varepsilon_{i} e_{i}$ and the coordinate asymmetry constant $\chi(B)$ is given by $\sup _{\varepsilon}\left\|g_{\varepsilon}\right\|$. Thus $\chi(E)=\inf \{\chi(B): B$ a basis for $E\}$ is the unconditional basis constant of $E$ defined in $\S 11$.

Theorem 2. If $\operatorname{dim} E=n,[A, \alpha] a$ Banach ideal, then

$$
n \leqq \alpha(E) \alpha^{\Delta}(E) \leqq 3 n(\delta(E))^{3} \text {. }
$$

A similar result for the parameter $\chi$ is a bit more difficult to state.

TheOrem 3. Let $\operatorname{dim} E=n, B=\left\{e_{i}\right\}_{i=1}^{n}$ a basis for $E$ and $[A, \alpha]$ a Banach ideal. For $J \subset\{1, \cdots, n\}$ let $E_{J}=\left[e_{i}: i \in J\right]$ and $a_{j}=\min \left\{\max \left(\alpha^{\Delta}\left(E_{I}\right): I \subset J\right)\right.$, card $J=j\}, j=1, \cdots, n$. Then

$$
\alpha(E) \leqq \chi(B)^{3} \sum_{i=1}^{n} a_{j}^{-1} .
$$

Application. Let $E \subset l_{p} \quad(1 \leqq p \leqq 2), \operatorname{dim} E=n$. Let $\mu(E)=\min \left\{S(E)^{2}\right.$, $\left.3(\delta(E))^{3}, 2(\chi(E))^{3}\right\}$. Then $K_{G} \mu(E) \sqrt{n} \geqq \pi_{1}(E) \geqq \sqrt{n}$ where $K_{G}$ is the Grothendieck constant. If $2<q<s<\infty$ and $E \subset l_{q}, \operatorname{dim} E=n$, then there is a constant $C_{q, s}$ such that

$$
\sqrt{n} \leqq \Pi_{1}(E) \leqq C_{q, s} \mu(E) n^{1-1 / s} .
$$

From these facts we obtain the result that for

$$
E_{n}=l_{p}^{n} \oplus l_{q}^{n}, \quad 1 \leqq p \neq q \leqq \infty, \quad \delta\left(E_{n}\right) \rightarrow \infty .
$$

We now give some slightly deeper results alluded to in our section on LUST.

THEOREM 4. Let $J_{n}$ (respectively, $I_{n}$ ) be the natural inclusion of $l_{2}^{n} \hat{\otimes} l_{2}^{n}$ (respectively, $\left.l_{2}^{n} \hat{\otimes} l_{2}^{n}\right)$ to $l_{2}^{n} \otimes^{\sigma} l_{2}^{n}\left(\equiv l_{2}^{n^{2}}\right)$. Then (1) $\nu_{1}\left(J_{n}\right) \sim n$ and $\pi_{1}\left(J_{n}\right) \sim n^{1 / 2}$; and, (2) $\nu_{1}\left(I_{n}\right) \sim n^{3 / 2}$ and $\pi_{1}\left(I_{n}\right) \sim n$ (for two sequences $\left(a_{n}\right)$ and $\left(b_{n}\right)$ with both $\left(a_{n} / b_{n}\right)$ and $\left(b_{n} / a_{n}\right)$ bounded, we write $\left.\left(a_{n}\right) \sim\left(b_{n}\right)\right)$.

THEOREM 5. Let $1 / p+1 / p^{\prime}=1$ and $1 / q+1 / q^{\prime}=1$. Then $\chi\left(l_{q^{n}}^{n} \hat{\otimes} l_{p^{\prime}}^{n}\right)=$ $\chi\left(l_{p}^{n} \otimes l_{q}^{n}\right) \sim n^{1 / 2}$ if $\infty \geqq q, \quad p \geqq 2 ; n^{1-1 / q} \quad$ if $p \geqq 2 \geqq q \geqq 1 ; n^{1-1 / p} \quad$ if $q \geqq 2 \geqq p \geqq 1$; $n^{3 / 2-1 / p-1 / q}$ if $2 \geqq p, q \geqq 1$.

ApPLications. (1) $\chi\left(l_{p}^{n} \dot{\otimes} l_{q}^{n}\right) \rightarrow \infty$;

(2) $l_{p} \hat{\otimes} l_{q}$ and $l_{q^{\prime}} \hat{\otimes} l_{p^{\prime}}$ are not isomorphic to a complemented subspace of a space with unconditional basis (problem of Kwapien-Pełczyński);

(3) the ideal $\Pi_{1}$ is not contained in the ideal $\Gamma_{1}$ (problem of Grothendieck); 
(4) there is an operator $T$ between certain Banach spaces $E$ and $F$ such that $T^{\prime} \in \Pi_{1}\left(F^{\prime}, E^{\prime}\right)$ and $T \notin I_{\infty}(E, F)$ (problem of Grothendieck); and

(5) there is an operator of the form $U V$ with $U^{\prime}, V \in \Pi_{1}$ yet $U V \notin I_{1}$ (problem of Grothendieck).

Concerning the weakly nuclear norm, D. R. Lewis [12.12] has recently obtained the following result.

THEOREM 5. Every finite dimensional space $E$ satisfies $\eta(E) \leqq 3 \delta(E)$.

From this result it follows that there is a sequence of finite dimensional spaces whose asymmetry constants are one but whose diagonal symmetry constants tend to $\infty$.

ApplicAtion. For each $n, n^{1 / 2} / 18 \leqq \delta\left(\mathscr{L}\left(l_{2}^{n}, l_{2}^{n}\right)\right) \leqq n^{1 / 2}$.

We now discuss projection constants. The projection constant $\lambda(E)$ is defined to be the infimum of the numbers $\lambda$ such that for every Banach space $Y \supset E$ there is a linear projection $P: Y \rightarrow E,\|P\| \leqq \lambda$. In the language of ideals $\lambda(E)=i_{\infty}(E)$. Let $c_{n}=\max \{\lambda(E): \operatorname{dim} E=n\}$. It is known that $c_{n} \leqq \sqrt{n}[\mathbf{1 2 . 1 0}]$.

Using ideal theory (which we suppress) it is possible to construct spaces with the largest known projection constant: Let $E(\alpha), 1<\alpha<n$, be the $n$-dimensional space of points $x=\left(x_{1} \cdots x_{n}\right)$ with

$$
\|x\|=\max \left\{\max _{1 \leqq i \leqq n}\left|x_{i}\right|, \alpha^{-1} \sum_{1}^{n}\left|x_{i}\right|\right\} .
$$

THEOREM 6. $\lambda(E(\sqrt{n}))=\left(n-\lambda\left(l_{1}^{n}\right)\right) /\left(2 \sqrt{n}-\lambda\left(l_{1}^{n}\right)-1\right) . \quad$ In particular $\lim _{n \rightarrow \infty} \lambda(E(\sqrt{n})) / \sqrt{n}=(2-\sqrt{2 / \pi})^{-1}$.

It turns out that $c_{2} \in\left[\frac{4}{3}, \sqrt{2}\right)$. Actually Gordon [12.2] shows that $c_{2}<1.414211$.

We now turn to the ideals $I_{p, q}$ and $J_{p, q}$. First we observe that when $p=q$, $I_{p, q}=\Gamma_{p}$ and $i_{p p}=\nu_{p}$.

THEOREM 7. (a) If $1 \leqq q \leqq p \leqq \infty$ then $j_{p q}\left(l_{2}^{n}\right)=\pi_{p}\left(l_{2}^{n}\right) \pi_{q^{\prime}}\left(l_{2}^{n}\right)$;

(b) if $1 \leqq q \leqq p \leqq \infty$ then $i_{p q}\left(l_{2}^{n}\right) \pi_{p}\left(l_{2}^{n}\right) \pi_{q^{\prime}}\left(l_{2}^{n}\right)=n$;

(c) if $1<q \leqq p<+\infty$ and $H$ is an infinite dimensional Hilbert space then

$$
i_{p q}(H)=2 \pi^{-1 / 2 p-1 / 2 q^{\prime}} \Gamma\left(\frac{p+1}{2}\right)^{1 / p} \Gamma\left(\frac{q^{\prime}+1}{2}\right)^{1 / p^{\prime}} .
$$

It turns out that $\nu_{p}(H)=i_{p p}(H)$ is the relative projection constant of Hilbert space embedded isometrically in the $L_{p}$-spaces.

Lacunary sequences in the $L_{p}$-spaces have been studied extensively. Indeed for any probability measure space $(\Omega, \Sigma, \mu)$ if $E$ is a closed subspace of $L_{p}(\mu)(2 \leqq p<\infty)$ isomorphic to a Hilbert space, then there is a constant $C_{E}$ such that $\|f\|_{p} \leqq C_{E}\|f\|_{2}$ for $f \in E$. Thus the following result is of some importance:

THEOREM 8. (a) Let $2 \leqq p \leqq \infty$; then

$$
\min _{\mu, H} \max _{f \in \mathbf{H}}\|f\|_{p} /\|f\|_{2}=i_{p_{2}}\left(l_{2}^{n}\right)
$$


where the minimum is taken over all subspaces $H \subset L_{p}(\mu)$ isometric to $l_{2}^{n}$ and probability measures $\mu$.

(b) Let $1 \leqq p \leqq 2$; then

$$
\min _{\mu, H} \max _{f \in \mathrm{H}}\|f\|_{2} /\|f\|_{p}=\pi_{p}\left(l_{2}^{n}\right) / n^{1 / 2}
$$

where the minimum ranges over all subspaces $H \subset L_{p}(\mu)$ isometric to $l_{2}^{n}$ complemented with $\nu_{\mathrm{p}}\left(l_{2}^{n}\right)$ norm projections, and probability measures $\mu$.

In particular if $\infty>p \geqq 2$, one obtains

$$
i_{p_{2}}\left(l_{2}\right)=2^{1 / 2}\left[\Gamma\left(\frac{p+1}{2}\right) / \pi^{1 / 2}\right]^{1 / p}=\inf _{\mu, H} \sup _{f \in H} \frac{\|f\|_{p}}{\|f\|_{2}}
$$

and for $1 \leqq p \leqq 2$,

$$
\inf _{\mu, H} \sup _{f \in H} \frac{\|f\|_{2}}{\|f\|_{p}}=2^{-1 / 2}\left(\pi^{1 / 2} / \Gamma\left(\frac{p+1}{2}\right)\right)^{1 / p} .
$$

See [12.11] for proofs of the above.

Theorem 5 admits a further application to projection constants:

Let $E$ be an $n$-dimensional Banach space. There is a constant $\lambda$ such that $d\left(E, l_{1}^{n}\right) \leqq \lambda i_{\infty}\left(E^{\prime}\right) \delta(E)^{3}$.

For a proof of this fact, see [12.12].

Finally we mention yet another result of Lewis [12.12].

THEOREM 9. For each odd integer $n \geqq 5$, there is an $n$-dimensional space $E_{n}$ with $\chi\left(E_{n}\right)>1$ and $\delta\left(E_{n}\right)=\eta\left(E_{n}\right)=1$.

We end this section with some further remarks on ideal parameters on Hilbert space. For proofs of these results, see [12.13]. Above we gave the values of $i_{p q}\left(l_{2}^{n}\right)$ and $i_{p q}\left(l_{2}\right)$. To present further results of this nature we introduce the following ideas. For $\alpha$ an ideal norm and $T: E \rightarrow F$, let $/ \alpha(T)=\alpha(T \phi)$ where $\phi: L_{1}(\mu) \rightarrow E$ is a surjection. The norm / $\alpha$ is called the left injective envelop of $\alpha$. Similarly the right injective envelop of $\alpha, \alpha \backslash$, is defined by $\alpha \backslash(T)=\alpha(J T)$, where $J: F \rightarrow L_{\infty}(\mu)$ is any isometric embedding. The injective envelop of $\alpha$ is $/ \alpha \backslash=/(\alpha \backslash)=(/ \alpha) \backslash$. Analogously, the left projective envelop of $\alpha, \backslash \alpha$, is defined by $\left\langle\alpha(T)=\alpha(U)\right.$ where $j_{F}: F \rightarrow F^{\prime \prime}$ is the canonical map, $U T=j_{F} T$ and $J: E \rightarrow L_{\infty}(\mu)$ is any isometric imbedding. Similarly, the right projective envelop of $\alpha, \alpha /$, is defined by $\alpha /(T)=\alpha(W)$ where $j_{F} T=\phi^{\prime \prime} W$ and $\phi: L_{1}(\mu) \rightarrow F$ is any surjection. The projective envelop of $\alpha$ is $|\alpha|=(\mid \alpha) /=\mid(\alpha /)$.

For $1 \leqq q \leqq p \leqq \infty, T: E \rightarrow F, S: F \rightarrow G$,

$$
\pi_{p^{\prime}}\left((S T)^{\prime}\right) \leqq / i_{p q}(T) \pi_{q^{\prime}}(S) \quad \text { and } \quad \pi_{1}\left((S T)^{\prime}\right) \leqq / \pi_{p}(T)_{p^{\prime}}^{i}(S) .
$$

Using this result one can prove

TheOREM 10 . Let $1 \leqq q \leqq p \leqq \infty$ and $H$ be a real Hilbert space.

(a) If $\operatorname{dim}(H)=n$,

$$
/ i_{p q}\left(l_{2}^{n}\right)=\pi_{p^{\prime}}\left(l_{2}^{n}\right) \pi_{q^{\prime}}\left(l_{2}^{n}\right)^{-1}, \quad / i_{q}\left(l_{2}^{n}\right)=\pi_{1}\left(l_{2}^{n}\right) \pi_{q^{\prime}}\left(l_{2}^{n}\right)^{-1},
$$


and

$$
/ \pi_{p}\left(l_{2}^{n}\right) \geqq n^{-1} \pi_{1}\left(l_{2}^{n}\right) \pi_{p}\left(l_{2}^{n}\right) .
$$

(b) If $\operatorname{dim} H=\infty$ and $q>1$, then

$$
/ i_{p q}(H)=\pi^{(p-q) / 2 p q} \Gamma\left(\frac{p^{\prime}+1}{2}\right)^{-1 / p^{\prime}} \Gamma\left(\frac{q^{\prime}+1}{2}\right)^{1 / q^{\prime}}
$$

and

$$
/ i_{q}(H)=\pi^{1 / 2 q} \Gamma\left(\frac{a^{\prime}+1}{2}\right)^{1 / q^{\prime}}
$$

(c) If $\operatorname{dim} H=+\infty$,

$$
/ \pi_{\mathrm{p}}(H) \geqq 2^{-1} \pi^{(p+1) / 2 p} \Gamma\left(\frac{p+1}{2}\right)^{-1 / p} .
$$

We remark that geometrically $/ i_{q^{\prime} p^{\prime}}\left(l_{2}\right)$ is a lower bound for the ratio of the $L_{p}$-norm and $L_{q}$-norm on infinite dimensional subspaces of $L_{q}(q \leqq p<\infty)$.

Moreover, the constant $/ \pi_{1}(H), \operatorname{dim} H=+\infty$, is just the Grothendieck constant $K_{G}$. While the exact value of this constant is still not known, $/ \pi_{p}(H)$ can be calculated for values of $p>1$.

FACTS. (1) For any $n \geqq 1, / \pi_{2}\left(l_{2}^{n}\right)=\pi_{1}\left(l_{2}^{n}\right) n^{-1 / 2}$ and $/ \pi_{2}\left(l_{2}\right)=\sqrt{\pi / 2}$.

(2) (Grothendieck). For any $n \geqq 1$,

Also,

$$
\mid \gamma_{2}^{*} \backslash\left(l_{2}^{n}\right)=\left(\pi_{1}\left(l_{2}^{n}\right)\right)^{2} n^{-1} \text { and } \mid \gamma_{2}^{*} \backslash\left(l_{2}\right)=\pi / 2 \text {. }
$$

$$
\mid \gamma_{2} /\left(l_{2}^{n}\right)=n\left(/ \gamma_{2}^{*} \mid\left(l_{2}^{n}\right)\right)^{-1} \text {. }
$$

(3) Let $H_{1}$ and $H_{2}$ be two infinite dimensional Hilbert spaces, $1 \leqq q \leqq p<\infty$ and $T \in \mathscr{L}\left(H_{1}, H_{2}\right)$. Then $\pi_{q}(T) \leqq / i_{q^{\prime} p^{\prime}}\left(l_{2}\right) \pi_{p}(T)$ and the constant $/ i_{q^{\prime} p^{\prime}}\left(l_{2}\right)$ is the best possible. In particular $([12.14]$ and $[12.15]), \pi_{1}\left(H_{1}, H_{2}\right)=\pi_{p}\left(H_{1}, H_{2}\right)$ for all $p \geqq 1$. (See Application 1, §8.)

13. Rademacher averages, type, cotype and stability. Let $\left(r_{j}\right)$ denote the Rademacher functions on $[0,1]$, i.e., $r_{j}(t)=\operatorname{sign} \sin 2^{j-1} 2 \pi t$. One form of the classical Khinchine inequality asserts that for $p \in[1, \infty)$ there are constants $A_{p}, B_{p}$ such that

$$
\begin{aligned}
B_{p}\left(\int_{0}^{1}\left|\sum_{j=1}^{n} a_{j} r_{j}(t)\right|^{2} d t\right)^{1 / 2} & \leqq\left(\int_{0}^{1}\left|\sum_{j=1}^{n} a_{j} r_{j}(t)\right|^{p} d t\right)^{1 / p} \\
& \leqq A_{p}\left(\int_{0}^{1}\left|\sum_{j=1}^{n} a_{j} r_{j}(t)\right|^{2} d t\right)^{1 / 2}
\end{aligned}
$$

for scalars $a_{1} \cdots a_{n}$.

By a result of Kahane [13.1] the above inequalities remain true for suitable constants $A_{p}, B_{p}$ if the scalars are replaced by elements in an arbitrary Banach space $E$ (and absolute values, of course, replaced by norms).

Definition. If $\left(x_{1}, \cdots, x_{n}\right)$ is a finite set of elements in a Banach space $E$ then the expression $\left(\int\left\|\sum x_{i} r_{j}(t)\right\|^{p} d t\right)^{1 / p}$ is called the $p$ th Rademacher average of the sequence $\left(x_{1}, \cdots, x_{n}\right)$.

We now present some fundamental work of Maurey [13.1]-[13.5] and 
Pisier [13.6]-[13.9]. (Let us mention that in this section we have been influenced by the lecture [13.10] of Pełczyński and the paper of Krivine [13.11].)

For $p \in(0,2]$ let

$$
\begin{aligned}
& p^{*}=p \text { if } p<2 \text {, } \\
& =\infty \text { if } p=2 \text {. }
\end{aligned}
$$

An operator $T \in \mathscr{L}(E, F)$ is said to be type p-Rademacher if there is an $r \in(0, \infty)$ and $C>0$ such that

$$
\left[\int\left\|\sum_{n=1}^{k} T\left(x_{n}\right) r_{n}(t)\right\|^{r} d t\right]^{1 / r} \leqq C\left(\sum_{n=1}^{k}\left\|x_{n}\right\|^{p}\right)^{1 / p} .
$$

An operator is said to be of type p-stable if there is a $C>0$ and $r \in\left(0, p^{*}\right)$ such that

$$
\left[\int\left\|\sum f_{n}(t) T\left(x_{n}\right)\right\|^{r} d t\right]^{1 / r} \leqq C\left(\sum\left\|x_{n}\right\|^{p}\right)^{1 / p} .
$$

Here $\left(f_{n}\right)$ is a stable sequence of order $p$ on $[0,1]$ i.e., there are $C_{n}>0$ and $p \in(0,2]$ such that $\int \exp \left(i f_{n}(t)\right) d t=\exp \left(-c_{n}|\lambda|^{1 / p}\right)$. A space $E$ is said to be of type p-Rademacher (type $p$-stable) if the identity mapping on $E$ is of type $p$-Rademacher (resp. type $p$-stable). It is clear what this says about Rademacher averages and the Khinchine inequality. If $p \in[2, \infty)$ then $E$ is said to be of cotype $p$-Rademacher if $\left(\sum_{i=1}^{n}\left\|x_{i}\right\|^{p}\right)^{1 / p}$ is dominated by some constant multiple of the 2-Rademacher average of the $x_{1} \cdots x_{n}$. We remark that Pełczyński [13.10] calls spaces of type 2 (cotype 2) spaces with subquadratic (superquadratic) Rademacher averages. We could use functions other than the Rademacher functions (e.g. the Gaussian averages) but we choose not to do this. Thus we will say type $p$ for type $p$-Rademacher; similarly for cotype $p$.

Finally a Banach space $E$ is of infratype $p, p \in(0,2]$, if there is a constant $C$ such that for all $x_{n} \in E$

$$
\inf _{\varepsilon_{n}= \pm 1}\left\|\sum \varepsilon_{n} x_{n}\right\| \leqq C\left(\sum\left\|x_{n}\right\|^{p}\right)^{1 / p} .
$$

Some remarks relating these concepts are in order.

(i) If $T$ is of type $p$ then $T$ is of type $q$ for any $q \leqq p$.

(ii) Subspaces and quotients of spaces of type $p$ (resp. type $p$-stable) are of type $p$ (type $p$-stable).

(iii) Subspaces of spaces of cotype $p$ are of cotype $p$, but cotype, in general, is not preserved by quotients.

(iv) $L_{p}(\mu)$ is of cotype 2 for $1 \leqq p \leqq 2$ and any measure $\mu$.

(v) If $p \in[1,2], L_{p}(\mu)$ is $q$-stable for $q \in(0, p)$. If $p \in[2, \infty), L_{p}(\mu)$ is $q$-stable for $q \in[0,2]$.

(vi) If $p \in(0,2]$ and $E$ is $p$-stable then $E$ is $q$-stable for $q \in(0, p]$.

(vii) If $p \in(1,2]$ and $E$ is $p$-stable then $E$ is of type $p$; the converse is false. Indeed $l_{p}$ is of type $p$ but not $p$-stable. 
(viii) If $E$ is of type $p, p \in(0,2]$, then $E$ is $q$-stable for $q<p$. In particular, for $p \in(0,2], L_{p}(\mu)$ is $q$-stable for $q \in(0, p)$ and if $p \in[2, \infty), L_{p}(\mu)$ is $q$-stable for $q \in[0,2]$.

(ix) If $p \in(0,2]$ and $E$ is $p$-stable then $E$ is $q$-stable for $q \in(0, p]$.

(x) If $E$ is of type $p$ then $E$ is of infratype $p$.

(xi) If $E$ is of type $p$ then $E^{\prime}$ is of cotype $p^{\prime}$. However, $l_{1}$ is of cotype 2 and $l^{\infty}$ is not of type $p$ for any $p>1$.

(xii) If $E$ is 1 -stable then there is a $p \in[2, \infty)$ such that both $E$ and $E^{\prime}$ are of cotype $p$.

We should mention that there are nontrivial examples of the various types of spaces discussed above. Part 2 below is due to N. Tomczak-Jaegermann [13.12]. For part 1 , see [13.13].

THEOREM 1. (1) If $p \in[1,2]$ every subspace of an $\mathscr{L}_{\mathrm{p}}$-space is of cotype 2 ; if $p \in[2, \infty)$ every subspace and quotient space of an $\mathscr{L}_{\mathrm{p}}$-space is of type 2 . 2 .

(2) If $1 \leqq p \leqq 2$, then $\sigma_{p}(H)$ is of cotype 2 ; and, if $2 \leqq p<\infty, \sigma_{p}(H)$ is of type

Of course, as we have seen, the spaces $\sigma_{p}(H), p \neq 2$, lack LUST.

We now consider the relationships of these concepts to ideal theory.

THEOREM 2. (a) If $E$ is of cotype 2 then $2 \in I_{E}$. In particular if $E$ is an $\mathscr{L}_{1, \lambda}$-space there is a constant $C_{p}$ such that $\pi_{p}(T) \leqq \lambda C_{p} \pi_{2}(T)$ for any $T \in$ $\mathscr{L}(E, F)$. If $E$ is an $\mathscr{L}_{\infty, \lambda}$-space and $1 \leqq p \leqq 2, \mathscr{L}\left(E, L_{p}(\mu)\right) \subset \Gamma_{2}\left(E, L_{p}(\mu)\right)$.

(b) If $F$ is of cotype 2 and $T \in \Pi_{q}(E, F)$ for some $q<\infty$ then $T \in \Pi_{2}(E, F)$.

A Banach space $E$ has the Grothendieck property [13.10] if $\mathscr{L}\left(c_{0}, E\right)=\pi_{2}\left(c_{0}, E\right)$. Pełczyński has conjectured that a Banach space $E$ is of cotype 2 if and only if $E$ has the Grothendieck property. The following results are known concerning this conjecture.

THEOREM 3. If $E$ is of cotype 2 then $E$ has the Grothendieck property.

The proof of this result rests on a profound result due to Maurey [13.14] concerning certain ideals.

Lemma. If $X$ is of type 2 and $Y$ of cotype 2 then $\mathscr{L}(X, Y) \subset \Gamma_{2}(X, Y)$.

For a wide class of spaces the conjecture is proved.

THeOREM 4. If $X$ has LUST then $X$ is of cotype 2 if and only if $X$ has the Grothendieck property.

THEOREM 5. If X has LUST then $X$ is of type 2 if and only if $X^{\prime}$ has the Grothendieck property and $X^{\prime}$ is not a $\mathscr{D}_{1}$-space.

Another result related to ideals is the following

Proposition. Let $p \in(0,1)$. Then $E$ is 1 -stable if and only if there is a $C>0$ such that for any quotient $G$ of $E^{\prime}$ and any $T \in \mathscr{L}(G, F), F$ arbitrary, one has $\pi_{p}(T) \leqq C \pi_{1}(T)$.

In this connection we remark that if $p \in[1,2)$ and $E$ is of type $p$-stable, 
then $E$ is of type $p+\varepsilon$-stable for some $\varepsilon$ in $(0,2-p)$. Since a subspace $E$ of $L_{p}$ is of type $p$-stable if and only if $l_{p} \not \subset E$ the above result generalizes the theorem of Rosenthal given in $\$ 9$.

B-Convexity. In this subsection we consider the work of Pisier [13.8]. A Banach space $E$ is $B-(k, \varepsilon)$-convex (for $\varepsilon$ in $[0,1]$ ) provided for all $x_{1} \cdots x_{n}$ in $E$

$$
\inf _{\varepsilon_{i}= \pm 1}\left\|\sum_{i=1}^{k} \varepsilon_{i} x_{i}\right\| \leqq k(1-\varepsilon) \sup \left\|x_{i}\right\| .
$$

Then $E$ is $B$-convex if there is a $k$ and $\varepsilon \in[0,1]$ such that $E$ is $B-(k, \varepsilon)$ convex. Let

$$
\lambda_{k}(E)=\inf \left\{\lambda: \inf _{\varepsilon_{i}= \pm 1}\left\|\sum \varepsilon_{i} x_{i}\right\| \leqq \lambda k \sup \left\|x_{i}\right\|\right\} .
$$

If $E$ is such that $\lambda_{N}(E)=1 / N^{1 / p^{\prime}}$ for some $N>1$ and $p^{\prime} \in[1, \infty)$, then $E$ is of infratype $q$ for all $q<p\left(1 / p+1 / p^{\prime}=1\right)$.

THEOREM 6. A Banach space $E$ is $B$-convex if and only if there is a $p \in(1,2]$ such that $E$ is of infratype $P$. Moveover,

$$
\Lambda_{E}=\sup \{p: E \text { is of infratype } p\}=\lim _{n \rightarrow \infty} \frac{\log n}{\log n \lambda_{n}(E)} .
$$

For a Banach space $E$ let

and

$$
\mu_{n}(E)=\inf \left\{\mu:\left(\int\left\|\sum_{1}^{n} r_{i}(t) x_{i}\right\|^{2} d t\right)^{1 / 2} \leqq \mu_{n} \sup _{1 \leqq i \leqq n}\left\|x_{i}\right\|\right\}
$$

$$
\nu_{n}(E)=\inf \left\{\nu:\left(\int\left\|\sum_{1}^{n} r_{i}(t) x_{i}\right\|^{2} d t\right)^{1 / 2} \leqq \nu \sqrt{n}\left(\sum_{1}^{n}\left\|x_{i}\right\|^{2}\right)^{1 / 2}\right\} .
$$

Here $\left(r_{i}(t)\right)$ denotes the Rademacher functions. Clearly

and

$$
\lambda_{n}(E) \leqq \mu_{n}(E) \leqq \nu_{n}(E) \leqq 1
$$

$$
\mu_{n}(E) \geqq 1 / \sqrt{n}, \quad \nu_{n}(E) \geqq 1 / \sqrt{n} .
$$

Also, if $E$ is such that $\nu_{N}(E)=1 / N^{1 / p^{\prime}}$ for some $N>1$ and $p^{\prime} \in[2, \infty)$, then $E$ is of type $q$ for any $q<p$.

Proposition. Let $R_{E}=\sup \{p: E$ is of type $p\}$. Then

$$
R_{E}=\lim _{n \rightarrow \infty} \frac{\log n}{\log \left[n \nu_{n}(E)\right]} .
$$

THEOREM 7. The following statements about the Banach space $E$ are equivalent:

(1) $E$ is not a $\mathscr{D}_{1}$-space;

(2) $E$ is of infratype $p$ for some $p \in(1, \infty)$ (equivalently $\Lambda_{E}>1$ );

(3) $E$ is of type $p$ for some $p \in(1,2]$ (equivalently $R_{E}<1$ );

(4) $E$ is of type $1+\varepsilon$-stable for some $\varepsilon$ in $(0,1]$; and

(5) $E$ is of type 1-stable. 
Finally, let us observe that if $p \in(0,2)$ and there is a constant $C$ such that for all $n$ there are elements $x_{1}^{n} \cdots x_{n}^{n}$ in $E$ such that

$$
\left(\sum_{i=1}^{n}\left|a_{i}\right|^{p}\right)^{1 / p} \leqq\left\|\sum a_{i} x_{i}^{n}\right\| \leqq C \sum_{1}^{n}\left|a_{i}\right|
$$

for all scalars $\left(a_{\mathrm{i}}\right)$, then $E$ is not $p$-stable. Thus from results of R. C. James [13.15] we have

Theorem 8. Any uniformly convex space is of type p (type p-stable) for some $p>1$.

Also,

Corollary. A Banach space $E$ is of type 1-stable if and only if $E^{\prime}$ is of type 1-stable.

LATTICES. When a lattice structure is present, more information can be obtained. Thus let $L$ be a Banach lattice, $V \in \mathscr{L}(L, F), q$ real. Then $V$ is said to be of type $\leqq q$ if there is a $C$ such that

$$
\left(\sum\left\|V\left(x_{i}\right)\right\|^{q}\right)^{1 / q} \leqq C\left\|\left(\sum\left|x_{i}\right|^{q}\right)^{1 / q}\right\|_{L} .
$$

Let $1 \leqq p \leqq q<\infty$. Then $V$ is said to be of type $\leqq(p, q)$ if there is a $C$ such that for $x_{1} \cdots x_{n} \in L$

$$
\left(\sum\left\|V x_{i}\right\|^{q}\right)^{1 / q} \leqq C\left\|\left(\sum\left|x_{i}\right|^{p}\right)^{1 / p}\right\|_{L} .
$$

Let $K_{p, q}(v)$ be the smallest $C$ satisfying the above.

THEOREM 9. For a Banach lattice $L$ and $V \in \mathscr{L}(L, F)$ the following statements are equivalent:

(1) for any positive operator $T \in \mathscr{L}(C(K), L), \quad V T \in \Pi_{p, q}(C(K), X)$ and $\pi_{p, q}(V T) \leqq C\|T\| ;$ and

(2) $K_{p, q}(V) \leqq C$.

(Let us recall that an operator $T \in \Pi_{p, q}(E, F)$ if there is a constant $C$ such that

$$
\left(\sum_{i=1}^{n}\left\|T x_{i}\right\|^{q}\right)^{1 / q} \leqq C\left(\sup _{\|f\|=1} \sum_{1}^{n}\left|f\left(x_{i}\right)\right|^{p}\right)^{1 / p}
$$

for arbitrary $x_{1} \cdots x_{n} \in E$. In this case inf $C, C$ satisfying the above, is denoted by $\pi_{p, q}(T)$. We have suppressed a growing and interesting series of papers on $p, q$-summing operators!)

Corollary. (a) Let $1 \leqq p_{0}<q$. If $V \in \mathscr{L}(L, F)$ is of type $\leqq\left(p_{0}, q\right)$ then $V$ is also of type $\leqq(p, q)$ for all $p \in[1, q)$.

(b) If $V$ is of type $\leqq(p, q)$ it is also of type $\leqq r$ for any $r>q$.

We can now describe the lattices of cotype $q, q>2$.

THEOREM 10. Let $L$ be a Banach lattice, $q>2$ and $V \in \mathscr{L}(L, F)$. The following are equivalent:

(1) $V \in \Pi_{1, q}(L, F)$;

(2) $V$ is of type $\leqq(p, q)$ for any $p \in[1, q)$; 
(3) $V$ is of cotype $q$; and

(4) there is a constant $C$ such that if $x_{1} \cdots x_{n}$ are pairwise disjoint elements of $L$ then $\left(\sum\left\|V x_{i}\right\|^{q}\right)^{1 / q} \leqq C\left\|\sum x_{i}\right\|$.

Letting $\mathrm{V}$ be the identity on $L$ yields the following result.

THEOREM 11. For a Banach lattice $L$ the following are equivalent:

(1) $L$ is of cotype 2;

(2) $L$ is of type $\leqq 2$; and

(3) $\mathscr{L}(C(K), L)=\Pi_{2}(C(K), L)$.

We now give some dual results. An operator $V \in \mathscr{L}(E, L), L$ a Banach lattice, is of type $\geqq(q, p), 1 \leqq p \leqq q<\infty$, if there is a constant $C$ such that for $x_{1}, \cdots, x_{n} \in E$,

$$
\left\|\left(\sum\left|V x_{i}\right|^{q}\right)^{1 / q}\right\|_{L} \leqq C\left(\sum\left\|x_{i}\right\|^{p}\right)^{1 / p} .
$$

Clearly $V$ is of type $\geqq(q, p)$ if and only if $V^{\prime}$ is of type $\leqq\left(q^{\prime}, p^{\prime}\right)$.

Proposition. If an operator $U \in \mathscr{L}(E, L)$ is of type $\geqq(2, p)$ and $V \in$ $\mathscr{L}(L, F)$ is of some finite type, then VU is of type p.

THEOREM 12. Let $E$ be a Banach space such that $E^{\prime}$ is of cotype 2 and $F$ a Banach space of cotype 2. If $U \in \mathscr{L}(E, F)$ factors through a lattice, then $U$ factors through a Hilbert space.

Proposition. If $L$ is a Banach lattice then either $L$ is a $\mathscr{S}_{\infty}$-space, or there is a $q<\infty$ such that the identity on $L$ is $(1, q)$-summing.

Thus,

THEOREM 13. Let $X$ be a Banach space. The following are equivalent:

(a) $c_{0}$ is not finitely representable in $X$;

(b) the identity on $X$ is $(1, q)$-summing;

(c) there is an $r<\infty$ such that $\mathscr{L}(C(K), X)=\Pi_{r}(C(K), X)$; and

(d) there is a $q<\infty$ such that $X$ is of cotype $q$.

THEOREM 14. Let $E$ be a space with LUST and $q>2$. The following are equivalent:

(a) $E$ is of cotype q; (b) the identity on $E$ is $(1, q)$-summing and (c) E has the Grothendieck property.

COROLlary. If E has LUST and $c_{0}$ is not finitely representable in $E$ then $E$ is of type $p$ if and only if $E^{\prime}$ is of cotype $p^{\prime}$.

14. Basis theory and ideals of operators on Hilbert space. Holub in a series of three papers $[\mathbf{1 4 . 1}]-[14.3]$ reexamined the fundamental work of Calkin, Schatten and Gohberg and Kreĭn and showed that many problems concerning ideals of operators on Hilbert space could be restated (and solved) in the standard language of Schauder bases and Köthe sequence spaces.

Of particular interest are the minimal ideals introduced by Schatten and the duals of such ideals. An ideal is minimal if it is the completion of $l_{2} \otimes l_{2}$ 
with respect to some unitarily invariant cross norm. (See [14.2] and the references there for appropriate definitions.) If $\alpha$ denotes such a cross norm on $l_{2} \otimes l_{2}$ it generates a symmetric gauge function $\psi$ on the finitely nonzero sequences by defining

$$
\psi\left(a_{i}, \cdots, a_{n}\right)=\left\|\sum_{i}^{n} a_{i} \varphi_{i} \otimes \psi_{i}\right\|_{\alpha}
$$

for any orthonormal sets $\left(\varphi_{i}\right)$ and $\left(\psi_{i}\right)$. If $(\mu, \psi)$ denotes the completion of the finitely nonzero sequences under $\psi$ then the unit vectors $\left(e_{i}\right)$ form a basis for $(\mu, \psi)$ which is similar to $\left(\varphi_{i} \otimes \psi_{i}\right)$ in $l_{2} \otimes_{\alpha} l_{2}$.

While this correspondence was well known, Holub utilized properties of bases to settle several problems. We give a few of those results. A unitarily invariant cross norm is significant if every operator in $\left(l_{2} \otimes_{\alpha} l_{2}\right)^{*}$ is compact.

THEOREM 1. Let $\alpha$ be a unitarily invariant cross norm. The following are equivalent.

(a) $\alpha$ is significant;

(b) $\alpha$ is not equivalent to $\nu$ (the greatest cross norm=nuclear norm); and

(c) the basis $\left(e_{i}\right)$ for $(\mu, \psi)$ is weakly convergent to 0 .

THEOREM 2. If $\alpha$ and $(\mu, \psi)$ are as above then the basis $\left(e_{i}\right)$ for $(\mu, \psi)$ is boundedly complete if and only if $l_{2} \otimes_{\alpha} l_{2}=\left(l_{2} \otimes_{\alpha^{\prime}} l_{2}\right)^{*}$. The basis is shrinking if and only if $\left(l_{2} \otimes_{\alpha} l_{2}\right)^{*}=l_{2} \otimes_{\alpha^{\prime}} l_{2}$. (Recall that a basis $\left(x_{i}\right)$ with coefficient functionals $\left(f_{i}\right)$ is boundedly complete if $\sup _{n}\left\|\sum_{i=1}^{n} a_{i} x_{i}\right\|<+\infty$ implies $\sum_{i=1}^{\infty} a_{1} x_{i}$ converges, and is shrinking if $\left(f_{i}\right)$ forms a basis for $E^{\prime}$.

Thus, if $\alpha$ is a unitarily invariant cross norm, $\left(l_{2} \otimes_{\alpha} l_{2}\right)^{*}=l^{2} \otimes_{\alpha^{\prime}} l_{2}$ if and only if $\left(l_{2} \otimes_{\alpha} l_{2}\right)^{*}$ is separable.

Let us return to minimal ideals. Clearly an ideal is minimal if it contains no proper closed subspace which is a norm ideal in $\mathscr{L}(H)$. An ideal is maximal if it is not itself a closed proper subspace of another norm ideal. If $J$ is a maximal ideal then $J$, the closure of the finite rank operators under the ideal norm, is the unique minimal ideal contained in $J$. An ideal $I$ such that $\tilde{J} \subset \mathbf{I} \subset \mathbf{J}$ is called intermediate.

Consider the simplest pair of minimal-maximal ideals, i.e., $\mathscr{L}(H)$ and $K(H)$. The results of Calkin show that for this pair there is no intermediate norm ideal (indeed, no two-sided ideal). On the other hand Mitiagin [14.4] exhibited an intermediate ideal between a certain pair of minimal-maximal ideals. Holub showed the true picture with the following remarkable result.

THEOREM 3. Let $\stackrel{J}{J} J$ be a minimal-maximal pair of norm ideals with $J \neq \mathscr{L}(H)$. Then there exists an intermediate ideal between $\stackrel{J}{J}$ and $J$.

The idea of the construction, as above, is to reduce the problem to problems concerning bases in Köthe sequence spaces.

In our final section we will continue with applications of Banach space theory to ideal theory.

15. Loose ends. In this final section we mention some work on the geometry of certain ideals. 
The geometry of $\sigma_{p}(H) H$ a Hilbert space has been studied by C. A. McCarthy [15.1] who proved, among other things, that the modulus of convexity of $\sigma_{p}(H)$ was the same as that of $l_{p}$ for $1<p<\infty$. We have previously mentioned the work of $N$. Tomczak-Jaegermann [15.2] concerning Rademacher averages in $\sigma_{p}(H)$. Holub [15.3], [15.4] has discussed the subspaces and metric geometry (e.g. extreme points and smooth points) of the trace class operators $\sigma_{1}(H)$.

More recently J. Arazy and J. Lindenstrauss [15.5] have discussed several linear topological properties of $\sigma_{p}(H)$. They make some striking comparisons with known results concerning the structure of the $L_{p}$-spaces.

Many years ago Schatten observed that $K(H, H)$ is not a conjugate space. Of course, there are spaces (e.g. $E=l_{p}$ and $F=l_{q}, p>q>1$ ) for which $K(E, F)$ is reflexive. The following result of Saphar and Feder [15.6] gives a complete answer to the question raised by the above results.

THeOReM 1. Let $E$ and $F$ be reflexive Banach spaces. Then $K(E, F)$ is either reflexive or not conjugate.

It would be of considerable interest to ascertain if the isomorphic version of the theorem is also valid.

It is only fitting that we close with applications of Banach space theory to Banach ideals. We will mention only a profound result of Rosenthal [15.7] and two equally remarkable results of Johnson and Figiel [15.8] and Johnson [15.9].

Theorem 2 (Rosenthal). A Banach space $E$ contains $l_{1}$ if and only if in $E$ there is a uniformly bounded sequence $\left(x_{n}\right)$ such that no subsequence of $\left(x_{n}\right)$ is weakly Cauchy.

A sequence $\left(x_{n}\right)$ in $E$ is weakly Cauchy if $\left(f\left(x_{n}\right)\right)$ is a convergent sequence for each $F \in E^{\prime}$. A weakly Cauchy sequence need not be weakly convergent, e.g. the unit vectors in $c_{0}$.

We now give a result of Petczyński and Ovsepian [15.10] which connects this result with Banach ideals. First we make a definition: Let $\mu$ be a probability measure on a compact Hausdorff space $S$. A subspace $Z$ of $C(S)$ is fat with respect to $\mu$ if the natural injection $I_{\mu}: L_{\infty}(\mu) \rightarrow L_{2}(\mu)$ when restricted to $Z$ is not a compact operator.

THeOREM 3. For any Banach space $E$ the following are equivalent:

(a) E contains $l_{1}$;

(b) there is a 2-absolutely summing operator from $\mathrm{X}$ onto $l_{2}$;

(c) $\Pi_{2}\left(E, l_{2}\right) \backslash K\left(E, l_{2}\right) \neq \varnothing$; and

(d) for every isometric embedding $j$ of $E$ into $C(S)$ there is a probability measure $\mu$ on $S$ such that $j(E)$ is fat with respect to $\mu$.

The difficult step in the proof, (d) $\Rightarrow(a)$, follows from the Rosenthal theorem.

The same reasoning used to prove Theorem 3 yields the following observation of $\mathrm{Y}$. Gordon (private communication). 
THEOREM 4. Let $E$ be a Banach space. Then either

(a) $\Pi_{1}\left(E, l_{2}\right) \subset K\left(E, l_{2}\right)$; or

(b) E contains a complemented copy of $l_{1}$; or

(c) E lacks LUST.

We now show how a construction of Figiel and Johnson [15.8] solves a problem of A. Pietsch [15.11]. We begin with an interesting example of Johnson [15.9]. Let $\left(E_{n}\right)$ be a sequence of finite dimensional Banach spaces such that, for $\varepsilon>0$ and any finite dimensional Banach space $F$, there is an $m$ such that $d\left(E_{m}, F\right)<1+\varepsilon$. (A simple compactness argument yields the existence of such $E_{n}$.) Let $E=\left(\bigoplus E_{n}\right)_{l_{1}}$. Then the conjugate of any separable Banach space is isomorphic to a complemented subspace of $E^{\prime}$. Since there are separable conjugate spaces which fail the approximation property, $E^{\prime}$ fails the approximation property. However, it is not difficult to see that $E$ has the approximation property.

THEOREM 5 (FIGIEL AND Johnson). For each positive integer $n$ there is an equivalent renorming of $E,\|\cdot\|_{n}$, such that if $F_{n}=\left(E,\|\cdot\|_{n}\right)$ then $F_{n}$ does not have the bounded approximation property with constant less than $n$.

Application. An operator $T: E \rightarrow F$ is dualizable if $T$ factors through a conjugate space:

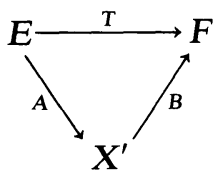

The dualizable norm $\delta(T)$ is defined by $\delta(T)=\inf \|A\|\|B\|$, the infimum taken over all such factorizations. If $\Delta$ denotes the class of all dualizable operators then $[\Delta, \delta]$ is a Banach ideal. Clearly $\|T\| \leqq \delta(T)$ for each $T \in \Delta$. A. Pietsch [15.11] has asked for examples of $T \in \Delta$ with $\|T\|<\delta(T)$.

THEOREM 6. For each integer $n$ there is an operator $T_{n} \in \Delta$ such that $\left\|T_{n}\right\|=1$ and $\delta\left(T_{n}\right) \geqq n$.

Indeed let $T_{n}$ denote the identity on $F_{n}$ of the Figiel-Johnson theorem. This operator meets the requirements.

There are at least two other interesting applications of the Johnson-Figiel construction. It is clear from the definition that the adjoint of a nuclear operator is also nuclear. The results of [15.8] show that the converse is false!

Also Pietsch [15.12] has defined $p$-integral operator via the following diagram:

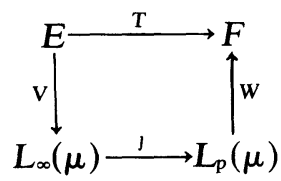

with meanings as in $\$ 5$. Again the Figiel-Johnson construction shows that the Pietsch definition and the Grothendieck definition given in $\$ 5$ are not the same. 


\section{BIBLIOGRAPHY}

1.1. J. Lindenstrauss and L. Tzafriri, The classical Banach spaces, Lecture Notes in Math., Springer-Verlag, Berlin and New York, 1973.

1.2. A. Pietsch, Theorie der Operatorenideale, Friedrich-Schiller-Universität, Jena 1972.

1.3. J. R. Retherford, Operator characterizations of $\mathscr{L}_{\mathrm{p}}$-spaces, Israel J. Math. 13 (1972), 337-347.

1.4. J. R. Retherford, D. R. Lewis and Y. Gordon, Banach ideals of operators with applications to the finite dimensional structure of Banach spaces, Israel J. Math. 13 (1972), 348-360.

1.5. - Banach ideals of operators with applications, J. Functional Analysis 14 (1973), $85-129$.

1.6. C. Stegall and D. R. Lewis, Banach spaces whose duals are isomorphic to $l_{1}(\Gamma), \mathrm{J}$. Functional Analysis 12 (1973), 177-187.

2.1. J. Lindenstrauss and A. Pełczyński, Absolutely summing operators in $\mathscr{L}_{\mathrm{p}}$-spaces and their applications, Studia Math. 29 (1968), 275-326. MR 37 \# 6743.

2.2. P. Enflo, A counterexample to the approximation problem in Banach spaces, Acta Math. 13 (1973), 309-317.

2.3. A. M. Davis, The approximation problem for Banach spaces, Bull. London Math. Soc. 5 (1973), 261-266.

2.4. J. S. Morrell and J. R. Retherford, p-trivial Banach spaces, Studia Math. 43 (1972), 1-25. MR $47 \# 2321$.

3.1. D. Hilbert, Grundzüge einer allgemeinen Theorie der linearen Integralgleichungen. IV, Nachr. Akad. Wiss. Göttingen. Math.-Phys. Kl. 1905 157-227.

3.2. E. Schmidt, Zur Theorie der linearen und nicht linearen Integralgleichungen, Math. Ann. 63 (1907), 433-466; ibid. 64 (1907), 161-174.

3.3. F. J. Murray and J. von Neumann, On rings of operators, Ann. of Math. 37 (1936), $116-229$.

3.4. J. von Neumann and R. Schatten, The cross-space of linear transformations. II, III, Ann. of Math. (2) 47 (1946), 608-630; (2) 49 (1948), 557-582. MR 8, 31; 10, 256.

3.5. J. W. Calkin, Two-sided ideals and congruences in the ring of bounded operators in Hilbert space, Ann. of Math. (2) 42 (1941), 839-873. MR 3. 208.

3.6. R. Schatten, Norm ideals of completely continuous operators, Ergebnisse der Mathematik und inhr Grenzgebiete, N.F., Heft 27, Springer-Verlag, Berlin, 1960. MR 22 \#9878.

3.7. 1. C. Gohberg and M. G. Krěnn, Introduction to the theory of linear nonselfadjoint operators in Hilbert space, "Nauka", Moscow, 1965; English transl., Transl. Math. Monographs, vol. 18, Amer. Math. Soc., Providence, R. I., 1969. MR 36 \#3137; 39 \# 7447.

3.8. W. Oosterbrink, Normed ideals, Dissertation, Univ. of Groningen, 1970.

3.9. F. Riesz, Über lineare Funktionalgleichungen, Acta Math. 41 (1918), 71-98.

3.10. S. Kakutani, Iteration of linear operations in complex Banach spaces, Proc. Imp. Acad. Tokyo 14 (1938), 295-300.

3.11. A. F. Ruston, On the Fredholm theory of integral equations for operators belonging to the trace class of a general Banach space, Proc. London Math. Soc. (2) 53 (1951), 109-124. MR 13, 138.

3.12. A. Grothendieck, Produits tensoriels topologiques et espaces nucléaires, Mem. Amer. Math. Soc. No. 16 (1955). MR 17, 763.

3.13. T. Kato, Perturbation theory for nullity, deficiency and other quantities of linear operators, J. Analyse Math. 6 (1958), 261-322. MR 21 \# 6541.

3.14. R. Schatten, A theory of cross-spaces, Ann. of Math. Studies, no. 26, Princeton Univ. Press, Princeton, N.J., 1950. MR 12, 186.

3.15. A. Pietsch, Absolut summierende Abbildungen in lokalkonvexen Räumen, Math. Nachr. 27 (1963), 70-103. MR 28 \#1473.

3.16. - Quasinukleäre Abbildungen in normierten Räumen, Math. Ann. 165 (1966), 76-90. MR 33 \#6412.

3.17. — Absolut p-summierende Abbildungen in normierten Räumen, Studia Math. 28 (1966/67), 333-353. MR 35 \#7162. 
3.18. - Hilbert-Schmidt-Abbildungen in Banach-Räumen, Math. Nachr. 37 (1968), 237-245. MR 38 \#2617.

3.19. - Ideal von Operatoren in Banachräumen, Mitt. Math. Ges. DDR (1968), 1-13.

3.20. - Ideale von $S_{\mathrm{p}}$-Operatoren in Banachräumen, Studia Math. 38 (1970), 59-69.

3.21. - $l_{\mathrm{p}}$-faktorisierbare Operatoren in Banachräumen, Acta Sci. Math. (Szeged) 31 (1970), 117-123. MR 42 \#889.

3.22. - Adjungierte normierte Operatorenideale, Math. Nachr. 48 (1971), 189-211. MR $44 \# 7307$.

4.1. B. S. Brudovskiī, Associated nuclear topology, mappings of type s, and strongly nuclear spaces, Dokl. Akad. Nauk SSSR 178 (1968), 271-273=Soviet Math. Dokl. 9 (1968), 61-63. MR 37 \#1952.

4.2. E. Dubinsky and M. S. Ramanujan, Inclusion theorems for absolutely $\lambda$-summing maps, Math. Ann. 192 (1971), 177-190. MR 45 \#2371.

4.3. - $\lambda$-nuclear spaces, Mem. Amer. Math. Soc. No. 128 (1972).

4.4. A. Pietsch, Nukleare lokalkonvexe Räume, Akademie-Verlag, Berlin, 1965. MR 31 \#6114.

4.5. L. Schwartz, Séminaire, Applications radonifiantes, Paris, 1969/70.

4.6. - Mésures cylindriques et applications radionifiantes dans les espaces du suites, Proc. Internat. Conf. on Functional Analysis and Related Topics (Tokyo, 1969), Univ. of Tokyo Press, Tokyo, 1970, pp. 41-59. MR 44 \#6932.

4.7. — Applications p-radonifiantes et théorème de dualité, Studia Math. 38 (1970), 203-213. MR 46 \#321.

4.8. A. Badrikian, Séminaire sur les fonctions aléatoires linéaires et les mésures cylindriques, Lecture Notes in Math., vol. 139, Springer-Verlag, Berlin and New York, 1970. MR 43 \#4994.

5.1. Same as $[\mathbf{3 . 2 2}]$.

5.2. Same as $[1.5]$.

6.1. C. Stegall and J. R. Retherford, Fully nuclear and completely nuclear operators with applications to $\mathscr{L}_{1}$ and $\mathscr{L}_{\infty}$-spaces, Trans. Amer. Math. Soc. 163 (1972), 457-493.

6.2. Same as [1.6].

6.3. I. M. Gel'fand, Abstrakte Funktionen und lineäre Operatoren, Mat. Sb. 4 (1938), 235-286.

6.4. A. Pełczyński, On Banach spaces containing $L_{1}(\mu)$, Studia Math. 30 (1968), 231-246. MR 38 \# 521.

6.5. J. Lindenstrauss and H. P. Rosenthal, The $\mathscr{L}_{\mathrm{p}}$-spaces, Israel J. Math. 7 (1969), 325-349. MR 42 \# 5012.

6.6. J. Cohen, Absolutely p-summing, $p$-nuclear operators and their conjugates, Dissertation, University of Maryland, College Park, Md., 1969.

6.7. J. R. Holub, A characterization of subspaces of $L_{p}(\mu)$, Studia Math. 42 (1972), 265-270. MR 46 \#2402.

6.8. W. B. Johnson, Factoring compact operators, Israel J. Math. 9 (1971), 337-345. MR 44 $\# 7318$.

6.9. S. Kwapien, On operators factorizable through $L_{p}$-spaces, Bull. Soc. Math. France Mém. 31/32 (1972), 215-225.

6.10. D. R. Lewis, Integral operators on $\mathscr{L}_{\mathrm{p}}$-spaces, Pacific J. Math. 46 (1973), 451-456.

6.11. A. Persson, On some properties of $p$-nuclear and p-integral operators, Studia Math. 33 (1969), 213-222. MR 40 \#769.

6.12. Same as $[1.5]$.

6.13. D. R. Lewis and Y. Gordon, Banach ideals on Hilbert spaces, Studia Math. (to appear).

7.1. A. Grothendieck, Résumé de la théorie métrique des produits tensoriels topologiques, Bol. Soc. Mat. São Paulo 8 (1953), 1-79 (1956), MR 20 \# 1194.

7.2. Same [2.1].

7.3. A. Grothendieck, Sur certaines classes des suites dans les espaces de Banach et le théorème de Dvoretzky-Rogers, Bol. Soc. Mat. São Paulo 8 (1953), 81-110 (1956). MR 20 \#1195. 
7.4. B. Maurey, Une nouvelle demonstration d'un théorème de Grothendieck, Séminaire Maurey-Schwartz 1972/73. Exposé 22.

7.5. K. H. Hoffman, Banach spaces of analytic functions, Prentice-Hall Series in Modern Analysis, Prentice-Hall, Englewood Cliffs, N.J., 1962. MR 24 \# A2844.

7.6. Same as [2.4].

8.1. S. Kwapien, On a theorem of $L$. Schwartz and its applications to absolutely summing operators, Studia Math. 38 (1970), 193-201. MR 43 \#3822.

8.2. P. Saphar, Applications p décomposantes et p-absolument sommantes, Israel J. Math. 11 (1972), 164-179. MR 45 \# 5779.

8.3. — Une charactérisation des sous-espaces de $L_{\mathrm{p}}$ et ses applications, Séminaire Maurey-Schwartz 1972/73. Exposé 14.

8.4. Same as $[6.6]$.

8.5. D. J. H. Garling, Lattice bounding, radonifying and absolutely summing mappings, Proc. Cambridge Philos. Soc. 77 (1974), 327-333.

8.6. N. J. Neilson, On Banach ideals determined by Banach lattices and their applications, Dissertationes Math. 109 (1973).

9.1. E. Dubinsky, A. Pełczyński and H. Rosenthal, On Banach spaces $X$ for which $\Pi_{2}\left(\mathscr{L}_{\infty}, X\right)=\mathscr{L}\left(\mathscr{L}_{\infty}, X\right)$, Studia Math. 54 (1972), 617-648.

9.2. W. Orlicz, Über unbedingte Konvergenz in Funktionenräumen. I, Studia Math. 4 (1933), 33-37; II, Studia Math. 4 (1933), 41-47.

10.1. H. P. Rosenthal, On subspaces of $L_{\mathrm{p}}$, Ann. of Math. (2) 97 (1973), 344-373. MR 47 \#784.

10.2. J. Bretagnolle and D. Dacunha-Castelle, Application de l'étude de certaines formes linéaires aléatories au plongement d'espaces de Banach dans les espaces $L^{p}$, Ann. Sci. École Norm. Sup. (4) 2 (1969), 437-480. MR 42 \#839.

10.3. B. Maurey, Un lemma de H. P. Rosenthal, Séminaire Maurey-Schwartz 1972/73, Exposé 21.

10.4. Same as $[\mathbf{1 . 1}]$.

10.5. Same as $[\mathbf{2 . 1}]$.

11.1. Y. Gordon and D. R. Lewis, Absolutely summing operators and local unconditional structures, Acta Math. 133 (1974), 27-48.

11.2. A. Petczyński, Some new isomorphic properties of the Banach spaces of holomorphic functions $A$ and $H^{\infty}$ (to appear).

11.3. D. R. Lewis, An isomorphic characterization of the Schmidt class (to appear).

11.4. W. B. Johnson, On finite dimensional subspaces of Banach spaces with local unconditional structure, Studia Math. 51, (1974), 223-238.

11.5. T. Figiel, W. B. Johnson and L. Tzafriri, On Banach lattices and spaces having local unconditional structure with applications to Lorenz function spaces (to appear).

11.6. R. C. James, Some self-dual properties of normed linear spaces, Ann. of Math. Studies, no. 69, Princeton Univ. Press, Princeton, N.J., 1972, pp. 159-175.

11.7. - Super-reflexive Banach spaces, Canad. J. Math. 24 (1972), 896-904. MR 47 \#9248.

11.8. P. Enflo, Banach spaces which can be given an equivalent uniformly convex norm, Israel J. Math. 13 (1972), 281-288.

11.9. L. Tzafriri, On Banach spaces with unconditional bases, Israel J. Math. 17 (1974), 84-93.

11.10. M. M. Day, Normed linear spaces, 3rd ed., Springer-Verlag, Berlin and New York, 1972.

11.11 W. Johnson and L. Tzafriri, On the local structure of subspaces of Banach lattices (to appear).

12.1. Y. Gordon, On p-absolutely summing constants of Banach spaces, Israel J. Math. 7 (1969), 151-163. MR 40 \#3269.

12.2. - Asymmetry and projection constants of Banach spaces, Israel J. Math. 14 (1973), 50-62. MR 47 \#7388.

12.3. Y. Gordon and D. J. H. Garling, Relations between some constants associated with finite-dimensional Banach spaces, Israel J. Math. 9 (1971), 346-361. 
12.4. Same as [11.1].

12.5. Y. Gordon and D. R. Lewis, Absolutely summing, $L_{1}$ factorable operators and their applications, Bull. Amer. Math. Soc. 79 (1973), 1270-1273.

12.6. A. Pietsch. Absolutely p-summing operators in $L_{r}$-spaces. I, II, Séminaire GoulaouicSchwartz 1970/71.

12.7. V. I. Gurarǐ̆, M. I. Kadec and V. I. Macaev, On Banach-Mazur distance between certain Minkowski spaces, Bull. Acad. Polon. Sci. Sér. Sci. Math. Astronom. Phys. 13 (1965), 719-722. MR 32 \#8113.

12.8. Same as [1.5].

12.9. S. Kwapien and A. Pełczyński, The main triangle projection in matrix spaces and its applications, Studia Math. 34 (1970), 43-68.

12.10. M. I. Kadeć and M. G. Snobar, Certain functionals on the Minkowski compactum, Mat. Zametki 10 (1971), 453-457=Math. Notes 10 (1971), 694-696. MR 45 \#861.

12.11. Same as $[1.5]$.

12.12. Same as $[\mathbf{1 1 . 3}]$.

12.13. Same as $[6.13]$.

12.14. A. Pełczyński, A characterization of Hilbert-Schmidt operators, Studia Math. 28 (1966/67), 355-360. MR 35 \# 7163.

12.15. D. J. H. Garling, Absolutely p-summing operators in Hilbert space, Studia Math. 38 (1970), 319-331. MR 43 \#6769.

13.1. J.-P. Kahane, Some random series of functions, Heath, Lexington, Mass., 1968. MR 40 $\# 8095$.

13.2. B. Maurey, Probabilités cylindriques, type et ordre. Applications radonifiantes, Séminaire Maurey-Schwartz 1972/73, Exposé 1.

13.3. - Probabilités cylindriques stables sur les espaces $L^{p}, p>2$ et applications $d u$ théorème de dualité, Séminaire Maurey-Schwartz 1972/73, Exposé 5.

13.4. - Espaces de cotype $p, 0<p \leqq 2$, Séminaire Maurey-Schwartz 1972/73, Exposé 7.

13.5. - Type et cotype dans les espaces munis de structures locales unconditionelles, Séminaire Maurey-Schwartz 1973/74, Exposé 24-25.

13.6. G. Pisier, Sur les espaces qui ne contiennent pas de $l_{n}$, Séminaire Maurey-Schwartz, Annexe 1972/73.

13.7. — "Type" des espaces normes, Séminaire Maurey-Schwartz 1973/74, Exposé 3.

13.8. - Sur les espaces qui ne contiennent pas de $l_{n}^{\prime}$ uniformément, Séminaire MaureySchwartz 1973/74, Exposé 7.

13.9. — Une propriété du type p-stable, Séminaire Maurey-Schwartz 1973/74, Exposé 8.

13.10. A. Pełczyński, On unconditional bases and Rademacher averages, Lecture, Kent State University, 1973.

13.11. J. L. Krivine, Théorème de factorisation dans les espaces réticulés, Séminaire MaureySchwartz 1973/74, Exposé 22-23.

13.12. N. Tomczak-Jaegerman, The modulus of smoothness and convexity and the Rademacher averages of trace classes $S_{p}(1 \leqq p<\infty)$, Studia Math. 50 (1974), 163-182.

13.13. G. Nordlander, On sign-independent and almost sign-independent convergence in normed linear spaces, Ark. Mat. 4 (1962), 287-296. MR 25 \#4330.

13.14. B. Maurey, These, École Polytechnique, Paris, 1973.

13.15. R. C. James, Uniformly non-square Banach spaces, Ann. of Math. (2) 80 (1964), 542-550. MR 30 \#4139.

14.1. J. R. Holub, Schauder bases and norm ideals of compact operators, Proc. Amer. Math. Soc. 38 (1973), 343-348. MR 47 \#5560.

14.2. _ Schauder bases and norm ideals of compact operators. II, Indiana Univ. Math. J. 24 (1974), 555-564.

14.3. - A characterization of the norm ideals of compact operators on Hilbert space, J. Math. Anal. Appl. 50 (1975), 596-606.

14.4. B. S. Mitjagin, Normed ideals of intermediate type, Izv. Akad. Nauk SSSR Ser. Mat. 28 (1964), 819-832; English transl., Amer. Math. Soc. Transl. (2) 63 (1967), 180-194. MR 30 \#4142.

15.1. C. A. McCarthy, $c_{p}$, Israel J. Math. 5 (1967), 249-271. MR 37 \#735. 
15.2. Same as $[\mathbf{1 3 . 1 2}]$.

15.3. J. R. Holub, On subspaces of separable norm ideals, Bull. Amer. Math. Soc. 79 (1973), 446-448. MR 47 \#2432.

15.4. - On the metric geometry of ideals of operators on Hilbert spaces, Math. Ann. 201 (1973), 157-163. MR 48 \#4757.

15.5. J. Arazy and J. Lindenstrauss, Some linear topological properties of the spaces $C_{p}$ of operators on Hilbert space (to appear).

15.6. P. Saphar and M. Feder, Spaces of compact operators and their dual spaces, Israel J. Math. (to appear).

15.7. H. P. Rosenthal, A characterization of Banach spaces containing $l^{1}$, Proc. Nat. Acad. Sci. U.S.A. 71 (1974), 2411-2413.

15.8. $\mathrm{T}$ Figiel and $\mathrm{W}$. B. Johnson, The approximation property does not imply the bounded approximation property, Proc. Amer. Math. Soc. 41 (1973), 197-200.

15.9. W. B. Johnson, A complementary universal conjugate Banach space and its relation to the approximation problem, Israel J. Math. 13 (1972), 301-310 (1973). MR 48 \#4700.

15.10. A. Pełczyński and R. I. Ovsepian, The existence in every separable Banach space of a fundamental, total and bounded biorthogonal sequence and related constructions of uniformly bounded orthonormal systems in $L_{2}$, Séminaire Maurey-Schwartz 1973/74, Exposé 20.

15.11. Same as [1.2].

15.12. A. Pietsch, p-majorisierbare vektorwertige Masse, Wiss. Z. Fredrich-Schiller-Univ. Jena/Thüringen 18 (1969), Heft 2, 243-247. MR 41 \#8627.

Department of Mathematics, Louisiana State University, Baton Rouge, Louisiana 70803

INSTITUT FÜR ANGEWANDTE MATHEMATIK UND INFORMATIK DER UNIVERSITÄT BONN, 53 BonN, Wegelerstrasse 6, FEDERAL RePUBLIC OF GERMANY 